\title{
A review of domestic animal diseases within the Pacific Islands region
}

\author{
Aurélie Brioudes ${ }^{\mathrm{a}, *}$, Jeffrey Warner ${ }^{\mathrm{a}}$, Robert Hedlefs ${ }^{\mathrm{a}}$, Bruce Gummow ${ }^{\mathrm{a}, \mathrm{b}}$ \\ ${ }^{a}$ School of Veterinary and Biomedical Sciences, Faculty of Medicine, Health and Molecular Sciences, James \\ Cook University, Townsville 4811, QLD, Australia \\ ${ }^{\mathrm{b}}$ Department of Production Animal Studies, Faculty of Veterinary Science, University of Pretoria, South Africa \\ *Corresponding author at: School of Veterinary and Biomedical Sciences Faculty of Medicine, Health and \\ Molecular Sciences James Cook University, Townsville 4811, QLD, Australia; Tel: +61 (07) 47814071; fax: \\ +61(07) 47791526.E-mail; Aurelie.Brioudes@my.jcu.edu.au
}

\section{Graphical abstract}

\section{Summary:}

Knowledge of terrestrial animal diseases in Pacific Island countries and territories is scarce and obsolete. These islands constitute a significant tropical region of the world and the livestock populations on these islands are potential hot spot for emerging diseases.

\section{Pictogramme:}

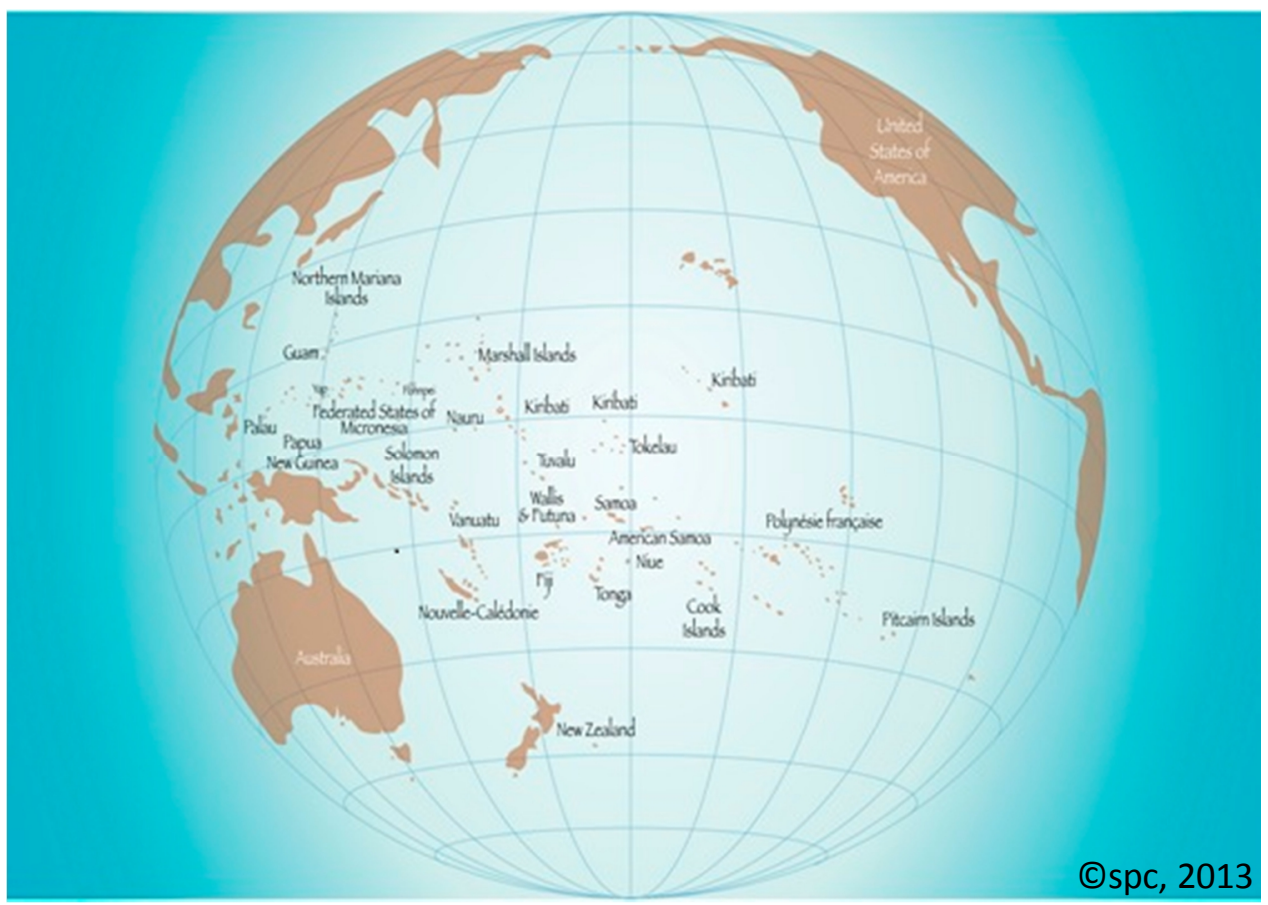

(Reproduced with permission from the Secretariat of the Pacific Community)

\section{Highlights:}

- Current information on animal diseases in Pacific Island countries and territories (PICTs) is scarce and no longer up to date.

- References retrieved from the peer-reviewed and gray literature over the last 20 years provide information on 116 diseases of domestic animals in 17 PICTs.

- 16 diseases or pathogens included in surveillance studies were not detected or reported as not present over 20 years of reporting by PICTs. 
- There is a need for more up-to-date studies on animal disease prevalence distribution to be carried out.

\begin{abstract}
The Pacific Island countries and territories (PICTs) are reported to be free of the most serious infectious livestock diseases which are prevalent in other parts of the globe, such as Highly Pathogenic Avian Influenza, Foot and Mouth Disease or Rabies. Yet there is a lack of scientifically based evidence to confirm this animal health status. This paper reviews what has been published on diseases of domestic animals in the Pacific Islands region with a particular focus on data from the last 20 years (1992-2012). Relevant published papers were identified by a computerized literature search of two electronic databases (PubMed and Web of Knowledge). The latest reports on the animal health situation submitted by the PICTs to the World Organisation for Animal Health (OIE) were accessed on the World Animal Health Information Database (WAHID) interface and included in this review. Additionally, paper searches of resources were undertaken at the library of the Secretariat of the Pacific Community (SPC) in Fiji to retrieve any relevant grayliterature for this review. The study eligibility criteria included qualitative or quantitative information on any disease (bacterial, viral, parasitic and other health disorders) affecting domestic terrestrial animals (mammals, reptiles, birds and bees) in any of the 22 PICTs members of the SPC. A total of 158 eligible references were retrieved of which only 77 (48.7\%) were published since 1992 and analysed in more details. One hundred and one diseases and pathogens were reported on for bee, bird, carabao, cat, cattle, crocodile, deer, dog, donkey, goat, horse, pig, pigeon, poultry and sheep in the Oceania region and in 17 PICTs in particular. The paper gives information about known animal diseases, their reported prevalence and diseases not reported within the Pacific Islands region. The study found retrieved literature on animal diseases in PICTs was scarce and no longer up to date. There is a need to improve the published knowledge on the current animal disease status in the region.
\end{abstract}

Keywords:

Pacific island; Domestic animal; Animal disease; Literature review.

\title{
1. Introduction
}

Emerging infectious diseases pose a major concern for animal health and have significant economic impact on the global livestock industry. These emerging diseases usually have no country boundaries and if they originate in a developing country could quickly spread to industrialized countries and other developing countries, and vice versa, mainly due to translocation of people and animals or through trade (Gummow, 2010). The tropical environment of Pacific Island Countries and Territories (PICTs), coupled with a close human, wild animal and domestic animal interface and the inter-island movement of people between PICTs all create situations that are conducive to the emergence of diseases (Gummow, 2010; Jones et al., 2008). However little has been published on what diseases of domestic animals occur within these islands or their prevalence.

The PICTs are said to be free of the most serious infectious livestock diseases which are prevalent in other parts of the world such as Highly Pathogenic Avian Influenza (HPAI), Classical Swine Fever (CSF), Foot and Mouth Disease (FMD) and Rabies (Angus, 1986; 
Newman and McKenzie, 1991; Secretariat of the Pacific Community, 2009b; Yarrow, 2008). But there appears to be a lack of scientifically based evidence to confirm this status. To date, the only known work which compiled information on animal diseases in Oceania is an annotated bibliography on animal husbandry and diseases in the Pacific area dating back 45 years (Pacific Science Information Center Bernice P. Bishop Museum Honolulu, 1966). Therefore a systematic review of papers compiling information on any diseases affecting domestic animals is warranted.

The objective of this study was to systematically review the current knowledge about the disease status of domestic animals in the Pacific Islands region, with a view to highlighting the gaps in knowledge and identifying the potential needs in terms of animal disease surveillance in this region.

\section{Methods}

A review was carried out on what has been published on diseases of domestic animals in the Pacific Islands region with a particular focus on data from the last 20 years (1992-2012). The review included relevant published papers identified by a computerized literature search of two electronic databases (PubMed and Web of Knowledge), reports on the animal health situation submitted by the PICTs to the World Organisation for Animal Health (OIE) and paper searches of resources at the library of the Secretariat of the Pacific Community (SPC) in Fiji. The SPC is an international organisation working in various domains, including agriculture and in particular animal health and production, to help Pacific Island people achieve sustainable development.

\subsection{Search strategy}

\subsubsection{PubMed and Web of Knowledge databases}

Peer-reviewed studies were sought in January 2013 on the PubMed and ISI Web of Knowledge databases using the following search strategy:

Search 1: (animal* OR livestock* OR herd* OR farm* OR cattle* OR bovine OR pig* OR swine OR sheep* OR ovine OR goat* OR caprine OR horse* OR equine OR chick* OR duck* OR bird* OR poultry OR bee OR bees OR apiculture* OR dog* OR cat* OR canine OR crocodile*)

Search 2: (health OR infection* OR sick* OR disease*OR zoono* OR outbreak* OR bacteria* OR virus* OR parasite* OR prevention OR control OR surveillance)

Search 3: "Pacific" OR “Oceania" OR "Micronesia" OR "Melanesia" OR "Polynesia" OR "American Samoa" OR "Cook Island" OR "Federated States of Micronesia" OR "Fiji" OR "French Polynesia” OR "Guam” OR "Kiribati” OR "Marshall Islands” OR "Nauru” OR "New Caledonia" OR "Niue" OR "Northern Mariana Islands" OR "Palau" OR "Papua New guinea" OR "Pitcairn Islands" OR "Samoa" OR "Solomon Islands" OR "Tokelau" OR "Tonga" OR "Tuvalu" OR "Vanuatu" OR "Wallis" OR "Futuna")

Search 1 AND Search 2 AND Search 3 
The "all fields" option in PubMed and "Topic" option in Web of Knowledge were used to allow retrieval of publications in which the search terms appeared in the titles or the abstracts or the keywords.

\subsubsection{Secretariat of the Pacific Community local database}

The gray literature (i.e. print and electronic formats that have not been formally published by commercial publishers) were reviewed by scrutinising the SPC library database and the electronic documents archived in the shared-drive of the Animal Health and Production team from the Land and Resources Division.

\subsubsection{WAHID interface}

All official animal health reports submitted by countries from Oceania to the World Organisation for Animal Health (OIE) were reviewed via the World Animal Health Information Database (WAHID) interface ( $\underline{\mathrm{OIE}})$. However, few PICTs are currently OIE member countries and/or report their animal health status. Hence data is only available on this database for Fiji, Federate States of Micronesia (FSM), New Caledonia, Papua New Guinea (PNG), Samoa, Tonga and Vanuatu. Moreover, some of these countries do not report consistently to OIE (some yearly reports are missing for some of the PICTs).

\subsection{Eligibility criteria}

\subsubsection{Inclusion criteria}

A publication was considered eligible for this review if it included qualitative or quantitative information on any disease (bacterial, viral, parasitic and fungal) affecting domestic terrestrial animals in any of the PICTs. Following the OIE definition, domestic terrestrial animals (mammals, birds and bees) are animals with "a phenotype selected by humans" and that "live under supervision or control by humans" (OIE, 2011b). The selection of the countries and territories to be included in this review is based on the official list of 22 PICTs members of the SPC and included American Samoa, Cook Islands, FSM, Fiji, French Polynesia, Guam, Kiribati, Marshall Islands, Nauru, New Caledonia, Niue, Northern Mariana Islands, Palau, PNG, Pitcairn Islands, Samoa, Solomon Islands, Tokelau, Tonga, Tuvalu, Vanuatu, and Wallis and Futuna.

\subsubsection{Exclusion criteria}

Experimental studies and studies on aquatic species were systematically excluded. Studies investigating zoonotic diseases in humans were included whenever data was also provided for domestic animals (even if the study focused on humans). Since this paper focused on domestic animals, references on wild animals were excluded unlessthe data was collected from captive native animals (birds in particular). Publications focusing on crocodile, deer and pigeon health were included as these animals are being farmed in PNG and New Caledonia.

When more than one reference was retrieved for the same study or related work between the different databases under different formats (publications, project reports, conference presentation), only one reference was kept (the most compressive one) to avoid duplication. 
The inclusion and exclusion criteria were applied to the title and abstract of all retrieved references. Considering the general literature search applied, the access to various databases of particular relevance for animal diseases in the Pacific Islands region and the ability of the author to review articles written in English and in French, which are the 2 most widely used languages in the studied area, this literature review probably includes most of the accessible references on the subject in the public domain.

\subsection{Data collection process}

The data collection process was undertaken in 2 steps. First, basic information was collected from all retrieved articles in order to assess which diseases have been reported in which PICTs. For this basic analysis, the following information was systematically recorded: the publication date, the country, the species, the disease, the type of causative agent (bacteria, virus, parasite, alga, toxins, tumour, fungi, rickettsiae), if the reference focus was on a zoonotic disease or not, and the type of study (case report, case series, review or survey). In a second step, considering that the objective of this review was to obtain a better understanding of the current animal disease situation in PICTs, only documents published or written in the last 20 years were selected to focus on the most recent information. A more detailed analysis of the key findings from these references was then performed by collecting additional data. When quantitative data was available, the time of the study, the number of animals tested and number of positive analyses were recorded to calculate the apparent prevalence of the disease. For references without quantitative data, the status of the disease was recorded using qualitative terminology (enzootic, present, clinical disease, outbreak notification etc).

\section{$3 \quad$ Results}

\subsection{Selected references}

The search strategy retrieved 6,336 publications on PubMed of which only 107 were considered relevant when applying the inclusion and exclusion criteria. Similarly, 3,585 publications were obtained initially from Web of Knowledge, and after discarding the overlapping references with PubMed, 12 extra references were selected making a list of 119 references selected from these two scientific literature databases. Additionally, a further 47 references were identified from the SPC local database and the OIE database for the Oceania

region. This database combines all the reports submitted by the PICTs between 2005 and 2011. For eight references, abstracts were not available and the full text document could not be retrieved. Moreover, one publication was written in German and had no abstract available in English. These references (see table 1) were thus excluded. A final list of 158 references was identified as matching the inclusion criteria from which only $77(48.7 \%)$ were published within the last 20 years (from 1992 to 2012) and were processed further. 
Table 1

References excluded from the literature review.

1. Anonymous (1946). CONTROL of infectious animal diseases in the South-West Pacific area. Vet. Rec. 58: 165.

2. Aslanian and Cheliadinova (1970). Current nosoareal of The distribution of brucellosis in the countries of Africa, Oceania. Zh. Mikrobiol. Epidemiol. Immunobiol. 47(5): 7277.

3. Jones (1976). The role of pigs in the dissemination of hookworm infections in Papua New Guinea. P. N. G. Med. 153-155.

4. Steele (1977). The zoonoses in the South Pacific and their significance. Int. J. Zoonoses 4(1): 1-20.

5. Fleury et al. (1985). Antibodies against paramyxoviruses 2 and 6 in birds from New Caledonia. Vet. Rec. 117(20): 530 .

6. Hellyar (1985). The introduction of brucellosis into the Trans. R. Soc. Trop. Med. Hyg. 79(4): 567-568.

7. Wernery and Schmidt (1985). Occurrence of enzootic Papua New Guinea. Dtsch. Tierarztl. Wochenschr. 92(5): 170-172.

8. Thevenon et al. (1989). Survey of contagious-diseases in apiaries. Recl. Med. Vet. 165(11): 899-903.

9. Bergin (1996). Parker Ranch: Pacific pioneer in animal health. Vet.

\subsection{Study characteristics}

As shown in Fig. 1, the number of references decreased over the years since the 1980's. Most of the references consist of surveys (65\%) and case series or case reports $(20 \%)$. Only one reference classified as a "review" was dedicated specifically to Papua New Guinea (Hide, 2003) while the remaining reviews are generally papers looking at a particular disease worldwide with little data provided for the Pacific Islands region. Among the 22 PICTs included in the eligibility criteria for this literature review, half of the references were providing data for PNG and for New Caledonia, with 35\% and 24\% of papers retrieved respectively for these 2 countries (Fig. 1). A quarter (25\%) of the references provided data on diseases for more than two animal species (coded as "multi species"), 18\% reported on diseases of cattle and $15 \%$ on pigs (Fig. 2). When looking at the agent involved in the diseases studied, almost half of the references $(46 \%$ with $n=235)$ reported on parasitic diseases, one quarter on bacterial diseases $(25 \%)$ and another quarter on viral diseases $(24 \%)$ (Fig.3). About $59 \%$ of the references (93/158) provided data on at least one zoonotic disease. The number of references published annually is very irregular. Peaks of publications were observed in 2004 and 2011(Fig.4). 


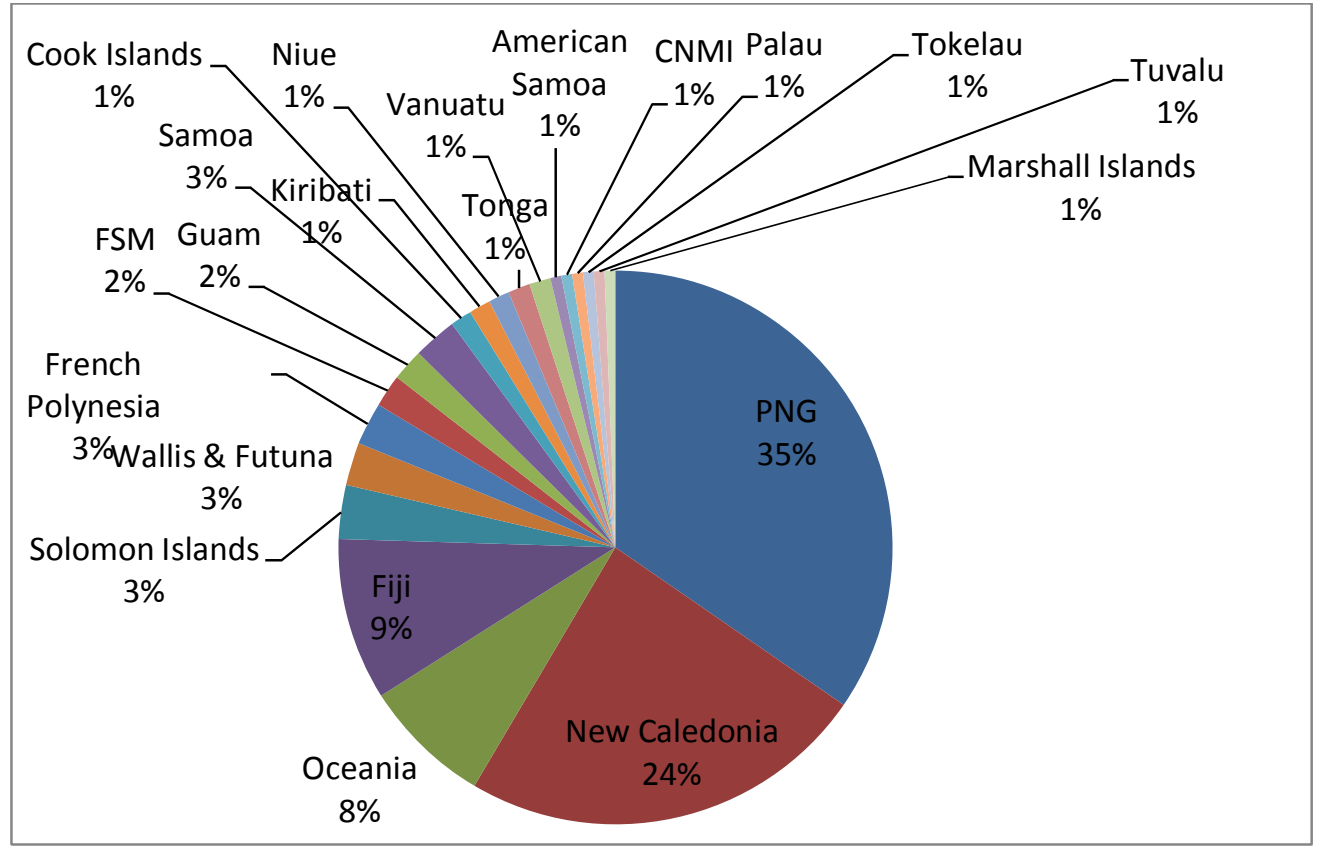

Fig. 1. Distribution of references sited per country in the Pacific Island countries and territories between 1992 and $2012(n=159)$

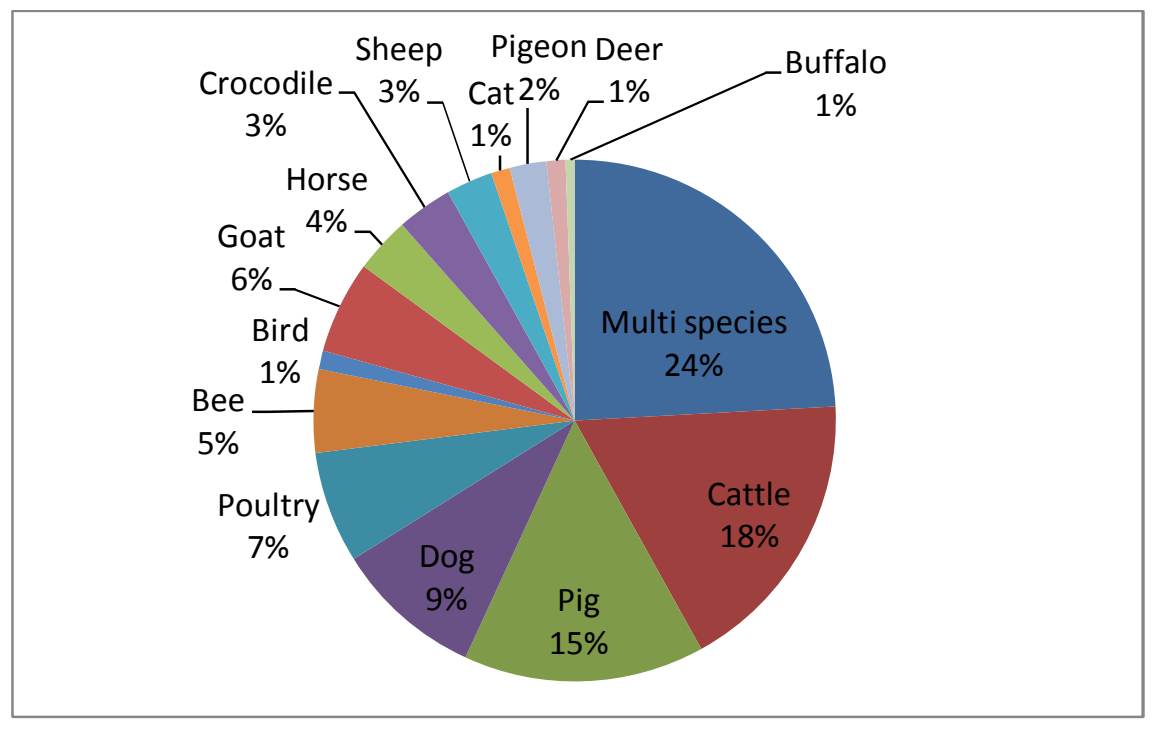

Fig. 2. Distribution of references sited per species in the Pacific Island countries and territories between 1992 and $2012(n=174)$ 


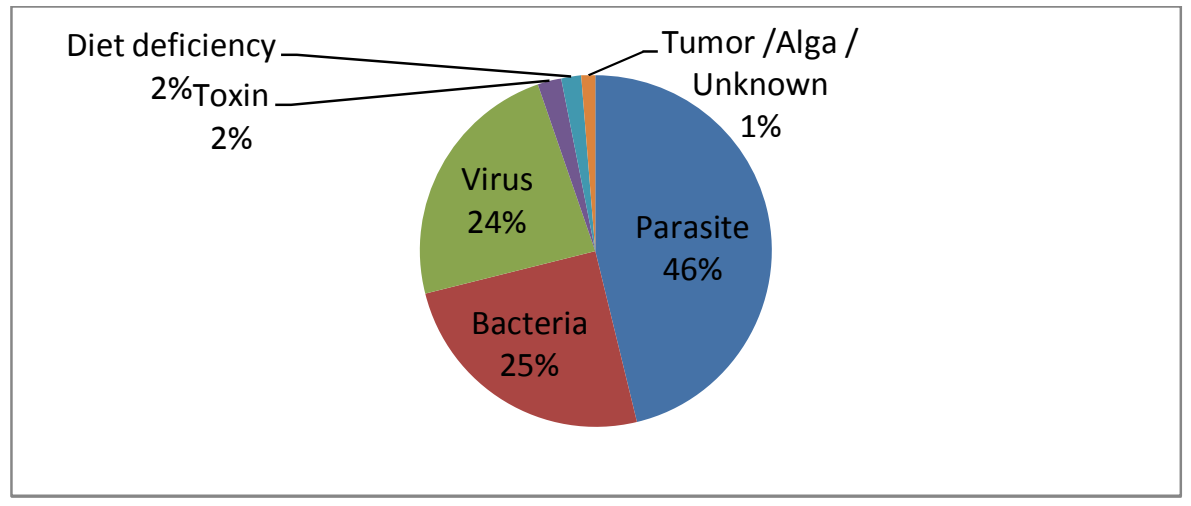

Fig. 3. Distribution of references sited per agent in the Pacific Island countries and territories between 1992 and $2012(n=225)$

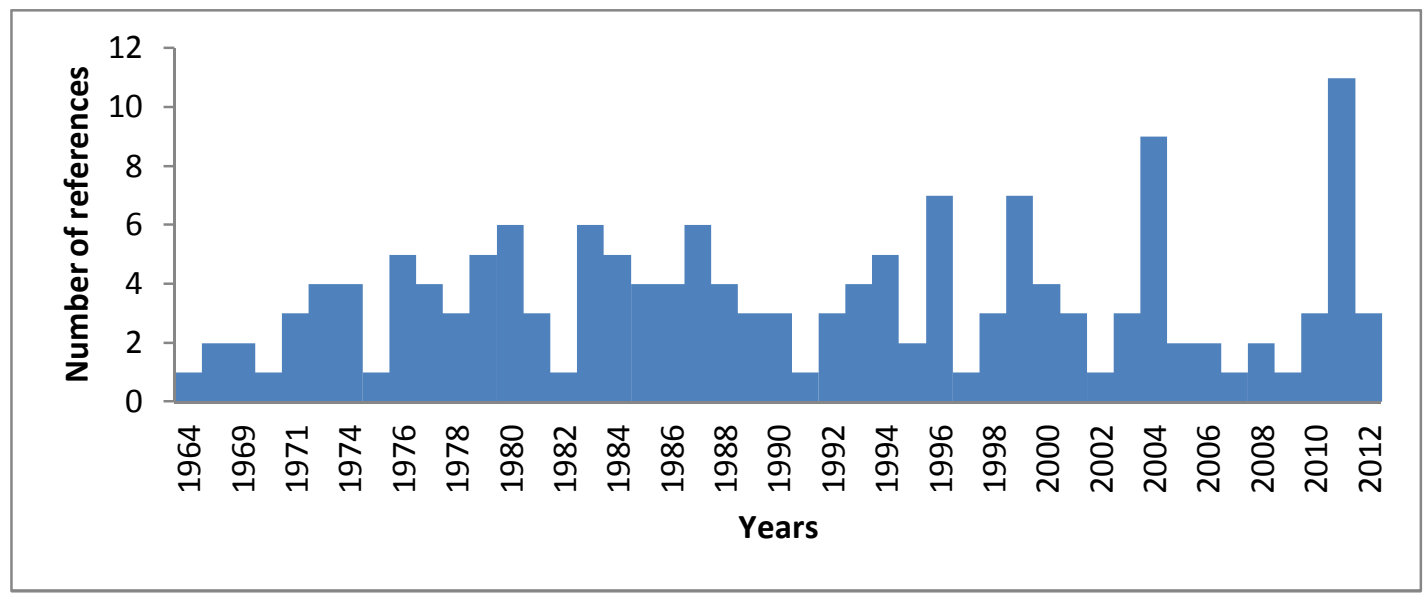

Fig.4. Distribution of references sited per year in the Pacific Island countries and territories between 1992 and $2012(n=158)$

\subsection{Results of individual studies from 1992 to 2012}

All the data on domestic animal diseases compiled from the 77 references between 1992 and 2012 for the Pacific Islands region are presented in Table 2 and Table 3. Table 2 shows a summary of data for diseases detected through surveys or reported as present. Table 3 gives the list of diseases not detected (based on negative laboratory results) or reported as not present (based on the absence of any clinical case observed) in the region. The dates presented in Tables 1 and 2 are the dates when the study was conducted, when this information was provided and the date of publication of the reference. The term "Oceania" is used for data provided for the Pacific Islands region in general without detailed specification of the affected countries.

The disease lists are presented with two categories of data: Quantitative data with the estimated prevalence of the studied animal disease and qualitative data with a description of the status of the animal disease in the specific PICTs. Categories of qualitative data include disease declared as not present or undetected ( - ); disease reported as present or detected $(+)$; 
Table 2

Animal diseases detected or reported as present in the Pacific Islands region based on the selected references from 1992 to 2012

\begin{tabular}{|c|c|c|c|c|c|}
\hline Disease & Species & Country & Date & Key result ${ }^{\mathrm{a}}$ & Reference \\
\hline \multicolumn{6}{|l|}{ BACTERIAL DISEASES } \\
\hline Actinomycosis & Multi species & French Polynesia & 2010 & + & (잍) \\
\hline \multirow{7}{*}{ American foulbrood } & Bee & Cook Islands & 1990 & 5.3 & $(\underline{\mathrm{SPC}}, 2004 \mathrm{a})$ \\
\hline & Bee & Fiji & 2001 & + & $(\underline{S P C}, 2004 b)$ \\
\hline & Bee & Fiji & 2011 & + & ( $\overline{\mathrm{OIE}}$ \\
\hline & Bee & French Polynesia & $2005-2007,2011$ & + & $(\overline{\mathrm{OIE}})$ \\
\hline & Bee & New Caledonia & $2005-2007,2011$ & + & $(\overline{\mathrm{OIE}})$ \\
\hline & Bee & Niue & 1998 & + & $(\underline{S P C}, 2004 c)$ \\
\hline & Bee & Tonga & 1991 & + & $(\overline{S P C}, 2004 \mathrm{e})$ \\
\hline \multirow[t]{6}{*}{ Anaplasmosis } & Carabao & Guam & 1999 & 14.3 & (Duguies et al., 2000) \\
\hline & Cattle & Guam & 1999 & 21.7 & (Duguies et al., 2000) \\
\hline & Cattle & Niue & 1992 & 1.5 & (Saville, 1996b) \\
\hline & Cattle & Samoa & 1997 & 3.2 & (Martin, 1999a) \\
\hline & Cattle & Solomon Islands & 1998 & 2.2 & (Martin and Epstein, 1999) \\
\hline & Dog & Samoa & $2010-2011$ & 6.1 & (Carslake et al., 2012) \\
\hline Anthrax & Multi species & PNG & 2011 & + & (OIE) \\
\hline \multirow[t]{11}{*}{ Avian encephalomyelitis } & Poultry & Cook Islands & 1993-1994 & 50.0 & (Saville, 1994) \\
\hline & Poultry & Fiji & 2008 & + & (OIE) \\
\hline & Poultry & French Polynesia & 2010 & + & $(\overline{\mathrm{OIE}})$ \\
\hline & Poultry & Guam & 1999 & 91.2 & (Duguies et al., 2000) \\
\hline & Poultry & Kiribati & 1992-1994 & 18.5 & (Saville, 1996a) \\
\hline & Poultry & Palau & 1996 & 22.0 & (Saville, 1999) \\
\hline & Poultry & Samoa & 1997 & 70.0 & (Martin, 1999a) \\
\hline & Poultry & Solomon Islands & 1998 & 35.9 & (Martin and Epstein, 1999) \\
\hline & Poultry & Tokelau & 1998 & 2.2 & (Martin, 1999b) \\
\hline & Poultry & Tonga & 1992-1994 & 42.9 & (Saville, 1996c) \\
\hline & Poultry & Wallis \& Futuna & 1997-1998 & 36.9 & (Martin, 1999c) \\
\hline \multirow[t]{3}{*}{ Bartonellosis } & Cat & New Caledonia & 2009 & + & (Mediannikov et al., 2011) \\
\hline & Cattle & New Caledonia & 2009 & + & (Mediannikov et al., 2011) \\
\hline & Deer & New Caledonia & 2009 & 31.0 & (Mediannikov et al., 2011) \\
\hline \multirow[t]{2}{*}{ Blackleg } & Multi species & Fiji & 2008 & + & (OIE) \\
\hline & Multi species & New Caledonia & 2009 & + & $(\overrightarrow{\mathrm{OIE}})$ \\
\hline \multirow[t]{3}{*}{ Botulism } & Multi species & French Polynesia & 2010 & + & $(\overline{\mathrm{OIE}})$ \\
\hline & Multi species & New Caledonia & 2010 & + & $(\overline{\mathrm{OIE}})$ \\
\hline & Poultry & Kiribati & 1992-1994 & + & (Saville, 1996a) \\
\hline \multirow[t]{2}{*}{ Bov. genital campylobacteriosis } & Cattle & New Caledonia & $2005-2007,2011$ & + & (OIE) \\
\hline & Cattle & Vanuatu & $2005-2007,2011$ & + & $(\overline{\mathrm{OIE}})$ \\
\hline \multirow[t]{12}{*}{ Brucellosis } & Cattle & Samoa & 1997 & 3.5 & (Martin, 1999a) \\
\hline & Multi species & Fiji & 2011 & + & (OIE) \\
\hline & Multi species & French Polynesia & 2006,2010 & + & $(\overline{\mathrm{OIE}})$ \\
\hline & Multi species & Tonga & 2011 & $+?$ & $(\overrightarrow{\mathrm{OIE}})$ \\
\hline & Pig & French Polynesia & 2005, 2007, 2011 & + & $(\overline{\mathrm{OIE}})$ \\
\hline & Pig & FSM & 2009 & + & $(\underline{\mathrm{OIE}})$ \\
\hline & Pig & PNG & 2011 & $+?$ & $(\underline{\mathrm{OIE}})$ \\
\hline & Pig & Tonga & 2011 & $+?$ & $(\overline{\mathrm{OIE}})$ \\
\hline & Pig & Tonga & 1992-1994 & 22.5 & (Saville, 1996c) \\
\hline & Pig & Wallis \& Futuna & 2004 & 7.1 & $(\overline{S P C}, 2004 f)$ \\
\hline & Pig & Wallis \& Futuna & 2007 & + & $(\overline{\mathrm{OIE}})$ \\
\hline & Pig & Wallis \& Futuna & 2011 & 34.0 & (Antras and Garin-Bastuji, 2011) \\
\hline Campylobacter contamination & Poultry & New Caledonia & $2005-2006$ & 96.7 & (Garin et al., 2012) \\
\hline \multirow[t]{5}{*}{ Chlamydiosis } & Crocodile & PNG & 2008 & + & (Huchzermeyer et al., 2008) \\
\hline & Pigeon & New Caledonia & 1992 & 7.4 & (Thevenon et al., 1992) \\
\hline & Poultry & New Caledonia & $2005-2007,2011$ & + & (OIE) \\
\hline & Poultry & Tonga & 2010 & $+?$ & $(\overline{\mathrm{OIE}})$ \\
\hline & Sheep & New Caledonia & $2005-2008$ & + & $(\overline{\mathrm{OIE}})$ \\
\hline \multirow[t]{5}{*}{ Clostridial infections } & Goat & Guam & 1999 & + & (Duguies et al., 2000) \\
\hline & Goat & Kiribati & 1992-1994 & + & (Saville, 1996a) \\
\hline & Poultry & Fiji & 2008 & + & (OIE) \\
\hline & Poultry & French Polynesia & 2010 & + & $(\underline{\mathrm{OIE}})$ \\
\hline & Poultry & New Caledonia & 2010 & + & $(\underline{O I E})$ \\
\hline Commensal \& opportunistic bacteria & Bird & Guam & $1982-86$ & 54.7 & (Savidge et al., 1992) \\
\hline \multirow[t]{2}{*}{ Dermatophilosis } & Multi species & French Polynesia & 2005 & + & (OIE) \\
\hline & Multi species & New Caledonia & 2005 & + & $(\overrightarrow{\mathrm{OIE}})$ \\
\hline Enterotoxaemia & Multi species & Fiji & 2008 & + & $(\overline{\mathrm{OIE}})$ \\
\hline & Multi species & New Caledonia & 2010 & + & ( $\overline{\mathrm{OIE}})$ \\
\hline Enzootic pneumonia & Pig & Samoa & 1997 & + & (Martin, 1999a) \\
\hline & Pig & Solomon Islands & 1998 & + & (Martin and Epstein, 1999) \\
\hline European foulbrood & Bee & French Polynesia & $2005-2007$ & + & (OIE) \\
\hline
\end{tabular}




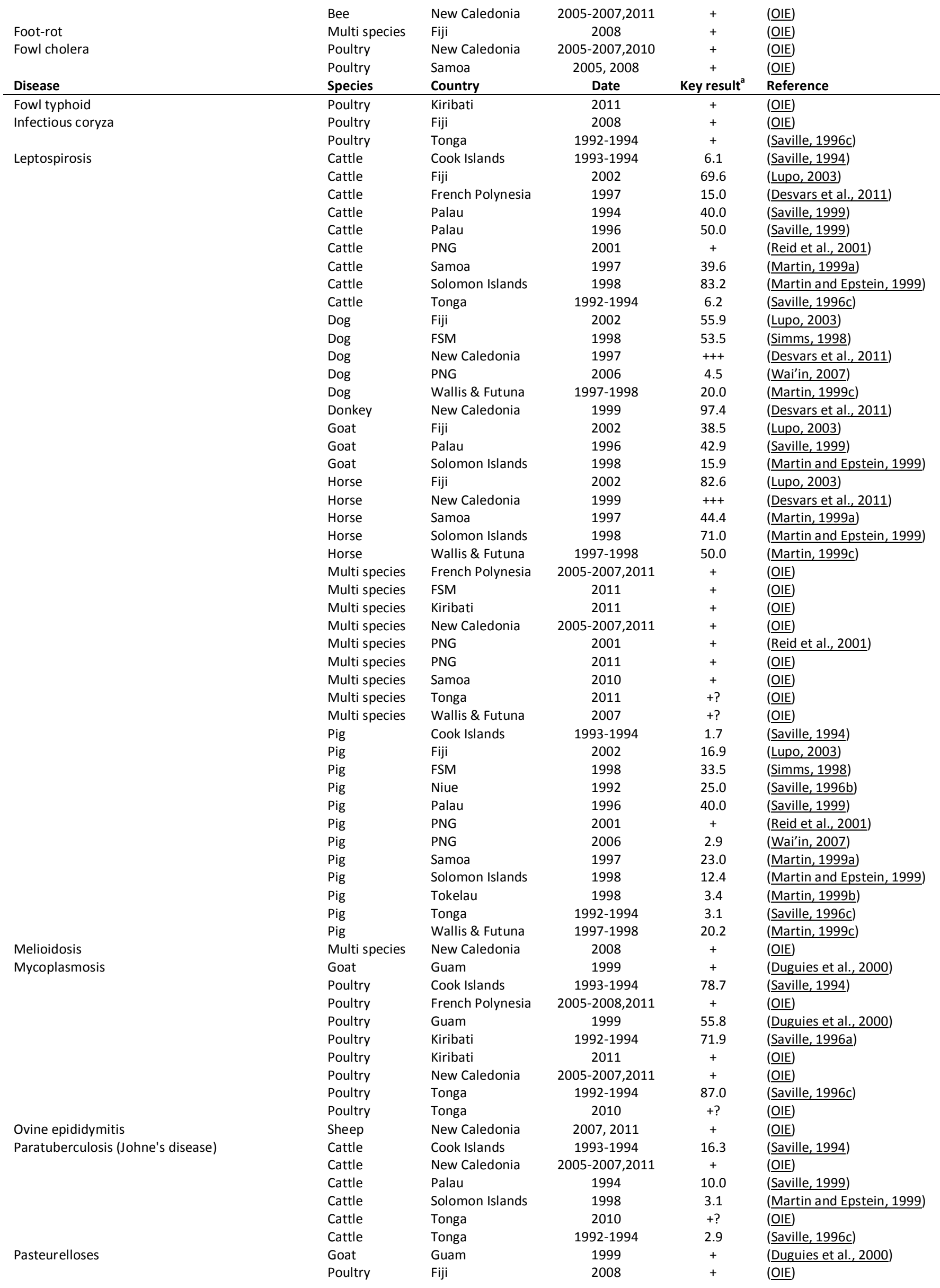




\begin{tabular}{|c|c|c|c|c|c|}
\hline & Poultry & French Polynesia & 2010 & + & (으) \\
\hline & Poultry & New Caledonia & 2010 & + & $(\underline{\mathrm{OIE}})$ \\
\hline \multirow[t]{7}{*}{ Salmonellosis } & Poultry & Cook Islands & 1993-1994 & 44.4 & (Saville, 1994) \\
\hline & Poultry & Fiji & 2008 & + & (OIE) \\
\hline & Poultry & French Polynesia & 2010 & + & (IE) \\
\hline & Poultry & Kiribati & 1992-1994 & 69.1 & (Saville, 1996a) \\
\hline & Poultry & New Caledonia & 2010 & + & (OIE) \\
\hline & Poultry & Tonga & 1992-1994 & 40.3 & (Saville, 1996c) \\
\hline & Poultry & Tonga & 2010 & $+?$ & ( $\underline{\mathrm{OIE}}$ ) \\
\hline Disease & Species & Country & Date & Key result ${ }^{a}$ & Reference \\
\hline Salmonellosis (Cont') & Sheep & New Caledonia & $2005-2007,2011$ & + & (OIE) \\
\hline \multirow[t]{4}{*}{ Serpulina pilosicoli } & Dog & PNG & 1997 & 5.3 & (Trott et al., 1997) \\
\hline & Dog & PNG & 1998 & + & (Trott et al., 1998) \\
\hline & Pig & PNG & 1997 & 17.0 & (Trott et al., 1997) \\
\hline & Poultry & PNG & 1997 & 50.0 & (Trott et al., 1997) \\
\hline Streptococcus suis type 2 & Pig & PNG & 1993 & 47.5 & (Paterson et al., 1993) \\
\hline \multirow[t]{5}{*}{ Swine erysipelas } & Pig & Fiji & 2008 & + & (을 \\
\hline & Pig & New Caledonia & 2010 & + & ( (OIE) \\
\hline & Pig & Samoa & 1997 & + & (Martin, 1999a) \\
\hline & Pig & Solomon Islands & 1998 & + & (Martin and Epstein, 1999) \\
\hline & Pig & Wallis \& Futuna & 1997-1998 & + & (Martin, 1999c) \\
\hline \multirow[t]{3}{*}{ Tetanus } & Goat & Cook Islands & 1993-1994 & + & (Saville, 1994) \\
\hline & Pig & Cook Islands & 1993-1994 & + & (Saville, 1994) \\
\hline & Pig & Samoa & 1997 & + & (Martin, 1999a) \\
\hline \multirow[t]{4}{*}{ Tuberculosis } & Cattle & Fiji & 2007,2011 & + & ( \\
\hline & Cattle & Samoa & 1997 & 1.4 & (Martin, 1999a) \\
\hline & Cattle & Tonga & 2011 & $+?$ & (OIE) \\
\hline & Poultry & Guam & 1999 & 28.6 & (Duguies et al., 2000) \\
\hline Vibrionic dysentery & Pig & New Caledonia & 2010 & + & (으) \\
\hline \multicolumn{6}{|l|}{ VIRAL DISEASES } \\
\hline Akabane virus & Cattle & Palau & 1995 & 30.0 & (Saville, 1999) \\
\hline \multirow{6}{*}{ Aujeszky's disease (Pseudorabies) } & Dog & Tonga & 1992-1994 & + & (Saville, 1996c) \\
\hline & Pig & Samoa & 1997 & 22.9 & (Martin, 1999a) \\
\hline & Pig & Tokelau & 1998 & 86.4 & (Martin, 1999b) \\
\hline & Pig & Tonga & 1992-1994 & 50.0 & (Saville, 1996c) \\
\hline & Pig & Tonga & 2011 & $+?$ & ( $\underline{\mathrm{OIE}}$ \\
\hline & Pig & Wallis \& Futuna & 1997-1998 & 8.5 & (Martin, 1999c) \\
\hline \multirow[t]{13}{*}{ Avian infectious bronchitis } & Poultry & Cook Islands & 1993-1994 & 24.1 & (Saville, 1994) \\
\hline & Poultry & French Polynesia & $2005-2007,2011$ & + & (OIE) \\
\hline & Poultry & Guam & 1999 & 49.3 & (Duguies et al., 2000) \\
\hline & Poultry & Kiribati & 1992-1994 & 38.8 & (Saville, 1996a) \\
\hline & Poultry & New Caledonia & 2005-2007,2011 & + & (OIE) \\
\hline & Poultry & Niue & 1991-93 & 38.9 & (Saville, 1996b) \\
\hline & Poultry & Palau & 1996 & 90.2 & (Saville, 1999) \\
\hline & Poultry & Samoa & 1997 & 96.7 & (Martin, 1999a) \\
\hline & Poultry & Samoa & 2005 & $+?$ & (으E) \\
\hline & Poultry & Solomon Islands & 1998 & 98.3 & (Martin and Epstein, 1999) \\
\hline & Poultry & Tokelau & 1998 & 100.0 & (Martin, 1999b) \\
\hline & Poultry & Tonga & 1992-1994 & 7.8 & (Saville, 1996c) \\
\hline & Poultry & Wallis \& Futuna & 1997-1998 & 88.7 & (Martin, 1999c) \\
\hline \multirow[t]{13}{*}{ Avian infectious laryngotracheitis } & Poultry & Cook Islands & 1993-1994 & 14.8 & (Saville, 1994) \\
\hline & Poultry & French Polynesia & 2007,2011 & + & (OIE) \\
\hline & Poultry & Guam & 1999 & 52.5 & (Duguies et al., 2000) \\
\hline & Poultry & Kiribati & 2011 & + & (OIE) \\
\hline & Poultry & New Caledonia & $2005-2008$ & + & (OIE) \\
\hline & Poultry & Palau & 1996 & 2.4 & (Saville, 1999) \\
\hline & Poultry & Samoa & 1997 & 7.7 & (Martin, 1999a) \\
\hline & Poultry & Samoa & 2005 & $+?$ & $\underline{(\mathrm{OIE})}$ \\
\hline & Poultry & Solomon Islands & 1998 & 50.0 & (Martin and Epstein, 1999) \\
\hline & Poultry & Tokelau & 1998 & 92.2 & (Martin, 1999b) \\
\hline & Poultry & Tonga & 1992-1994 & 3.9 & (Saville, 1996c) \\
\hline & Poultry & Tonga & 2010 & $+?$ & (OIE) \\
\hline & Poultry & Wallis \& Futuna & 1997-1998 & 27.5 & (Martin, 1999c) \\
\hline Avian leukosis & Poultry & Kiribati & 1992-1994 & + & (Saville, 1996a) \\
\hline \multirow[t]{2}{*}{ Black queen cell virus } & Bee & Niue & 1992, 1994 & +++ & (Saville, 1996b) \\
\hline & Bee & Solomon Islands & 1993 & 2.0 & (Reid and Van Eaton, 1993) \\
\hline \multirow[t]{3}{*}{ Bluetongue } & Cattle & Guam & 1999 & 8.3 & (Duguies et al., 2000) \\
\hline & Cattle & Solomon Islands & 1998 & 4.2 & (Martin and Epstein, 1999) \\
\hline & Multi species & PNG & 2011 & $+?$ & (을 \\
\hline Bovine virus diarrhea & Cattle & Cook Islands & 1993-1994 & 16.3 & (Saville, 1994) \\
\hline & Cattle & French Polynesia & 2006-2007,2011 & + & ( $\underline{\mathrm{OIE}})$ \\
\hline
\end{tabular}




\begin{tabular}{|c|c|c|c|c|c|}
\hline & Cattle & New Caledonia & 2006-2007,2011 & + & ( $\underline{\text { OIE }})$ \\
\hline & Cattle & Samoa & 1997 & 45.3 & (Martin, 1999a) \\
\hline & Cattle & Tonga & 1992-1994 & 8.2 & (Saville, 1996c) \\
\hline & Cattle & Tonga & 2011 & $+?$ & (OIE) \\
\hline Caprine arthritis encephalitis & Goat & Palau & 1996 & 16.7 & (Saville, 1999) \\
\hline \multirow[t]{3}{*}{ Chronic paralysis virus } & Bee & Niue & 1992, 1994 & + & (Saville, 1996b) \\
\hline & Bee & Samoa & 1996 & + & $(\underline{S P C}, 2004 d)$ \\
\hline & Bee & Solomon Islands & 1993 & +++ & (Reid and Van Eaton, 1993) \\
\hline Enterovirus encephalomyelitis & Pig & Cook Islands & 1993-1994 & + & (Saville, 1994) \\
\hline \multirow[t]{5}{*}{ Enzootic bovine leukosis } & Cattle & French Polynesia & 2005-2007, 2011 & + & ( $\underline{\text { OIE }}$ \\
\hline & Cattle & Guam & 1999 & 11.8 & (Duguies et al., 2000) \\
\hline & Cattle & Palau & 1994 & 44.4 & (Saville, 1999) \\
\hline & Cattle & Solomon Islands & 1998 & 2.7 & (Martin and Epstein, 1999) \\
\hline & Cattle & Tonga & 1992-1994 & 1.2 & (Saville, 1996c) \\
\hline Disease & Species & Country & Date & Key result ${ }^{a}$ & Reference \\
\hline \multirow{2}{*}{ Enzootic bovine leukosis (Cont') } & Cattle & Tonga & 2010 & $+?$ & (IE) \\
\hline & Cattle & Vanuatu & 2005 & $+?$ & $(\underline{\mathrm{OIE}})$ \\
\hline Equine influenza & Horse & Guam & 1999 & 25.2 & (Duguies et al., 2000) \\
\hline \multirow[t]{8}{*}{ Equine rhinopneumonitis } & Horse & Guam & 1999 & 67.9 & (Duguies et al., 2000) \\
\hline & Horse & New Caledonia & $2005-2007,2011$ & + & (OIE) \\
\hline & Horse & Samoa & 1997 & 36.5 & (Martin, 1999a) \\
\hline & Horse & Samoa & 2005 & + & $(\underline{\mathrm{OIE}})$ \\
\hline & Horse & Solomon Islands & 1998 & 71.0 & (Martin and Epstein, 1999) \\
\hline & Horse & Tonga & 1992-1994 & 1.1 & (Saville, 1996c) \\
\hline & Horse & Tonga & 2010 & $+?$ & 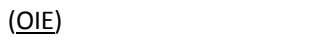 \\
\hline & Horse & Wallis \& Futuna & 1997-1998 & 16.7 & (Martin, 1999c) \\
\hline \multirow[t]{8}{*}{ Fowl pox } & Poultry & Cook Islands & 1993-1994 & + & (Saville, 1994) \\
\hline & Poultry & French Polynesia & 2005 & + & 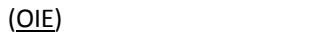 \\
\hline & Poultry & Kiribati & 1992-1994 & +++ & (Saville, 1996a) \\
\hline & Poultry & New Caledonia & 2005 & + & ( $\underline{\mathrm{OIE}}$ \\
\hline & Poultry & Palau & 1996 & + & (Saville, 1999) \\
\hline & Poultry & Samoa & 1997 & + & (Martin, 1999a) \\
\hline & Poultry & Samoa & 2005 & + & (OIE) \\
\hline & Poultry & Tonga & 1992-1994 & +++ & (Saville, 1996c) \\
\hline Getah virus & Horse & Oceania & 2000 & +++ & (Fukunaga et al., 2000) \\
\hline Hepatitis E virus & Pig & New Caledonia & 2011 & 6.5 & (Kaba et al., 2011) \\
\hline \multirow[t]{10}{*}{ Infectious bovine rhinotracheitis } & Cattle & French Polynesia & $2005-2007,2011$ & + & ( $\underline{\mathrm{OIE}})$ \\
\hline & Cattle & Guam & 1999 & 2.8 & (Duguies et al., 2000) \\
\hline & Cattle & New Caledonia & 1991 & 45.8 & (Vilain et al., 1994) \\
\hline & Cattle & New Caledonia & 2005-2007,2011 & + & 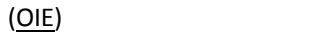 \\
\hline & Cattle & Palau & 1994 & 50.0 & (Saville, 1999) \\
\hline & Cattle & Samoa & 1997 & 68.7 & (Martin, 1999a) \\
\hline & Cattle & Solomon Islands & 1998 & 52.7 & (Martin and Epstein, 1999) \\
\hline & Cattle & Tonga & 1992-1994 & 18.1 & (Saville, 1996c) \\
\hline & Cattle & Tonga & 2010 & $+?$ & (OIE) \\
\hline & Cattle & Vanuatu & 2005 & $+?$ & ( \\
\hline \multirow[t]{17}{*}{ Infectious Bursal Disease (Gumboro) } & Poultry & Cook Islands & 1993-1994 & 37.1 & (Saville, 1994) \\
\hline & Poultry & Fiji & 2007 & $+?$ & ( \\
\hline & Poultry & Fiji & 2008 & + & ( $\underline{\text { OIE })}$ \\
\hline & Poultry & French Polynesia & $2005-2007,2011$ & + & ( $\underline{\text { OIE })}$ \\
\hline & Poultry & Guam & 1999 & 73.9 & (Duguies et al., 2000) \\
\hline & Poultry & Kiribati & 1992-1994 & 67.4 & (Saville, 1996a) \\
\hline & Poultry & Kiribati & 2011 & + & (잍) \\
\hline & Poultry & New Caledonia & 2005-2007,2011 & + & (밑) \\
\hline & Poultry & Niue & 1991-93 & 15.0 & (Saville, 1996b) \\
\hline & Poultry & Palau & 1996 & 12.2 & (Saville, 1999) \\
\hline & Poultry & PNG & 2011 & $+?$ & (잍) \\
\hline & Poultry & Samoa & 1997 & 22.5 & (Martin, 1999a) \\
\hline & Poultry & Solomon Islands & 1998 & 2.7 & (Martin and Epstein, 1999) \\
\hline & Poultry & Tokelau & 1998 & 72.2 & (Martin, 1999b) \\
\hline & Poultry & Tonga & 1992-1994 & 45.5 & (Saville, 1996c) \\
\hline & Poultry & Tonga & 2010 & $+?$ & ( $\underline{\text { OIE }}$ \\
\hline & Poultry & Wallis \& Futuna & 1997-1998 & 92.9 & (Martin, 1999c) \\
\hline \multirow[t]{2}{*}{ Japanese encephalitis virus } & Horse & Samoa & 1997 & 10.2 & (Martin, 1999a) \\
\hline & Multi species & PNG & 2011 & $+?$ & ( $\underline{\mathrm{OIE}}$ \\
\hline Kashmir bee virus & Bee & Solomon Islands & 1993 & 13.9 & (Reid and Van Eaton, 1993) \\
\hline Low pathogenic avian influenza & Poultry & PNG & 2011 & $+?$ & $(\underline{\mathrm{OIE}})$ \\
\hline Malignant catarrhal fever & Cattle & New Caledonia & $2005-2006$ & + & $(\overline{\mathrm{OIE}})$ \\
\hline \multirow[t]{3}{*}{ Marek's disease } & Poultry & Cook Islands & 1993-1994 & 25.9 & (Saville, 1994) \\
\hline & Poultry & French Polynesia & 2005-2007,2011 & + & ( $\underline{\text { OIE }}$ \\
\hline & Poultry & Guam & 1999 & 44.1 & (Duguies et al., 2000) \\
\hline
\end{tabular}




\begin{tabular}{|c|c|c|c|c|c|}
\hline & Poultry & Kiribati & 1992-1994 & 3.9 & (Saville, 1996a) \\
\hline & Poultry & New Caledonia & 2005-2007,2011 & + & (OIE) \\
\hline & Poultry & Niue & 1991-93 & 5.0 & (Saville, 1996b) \\
\hline & Poultry & Palau & 1996 & 46.3 & (Saville, 1999) \\
\hline & Poultry & Samoa & 1997 & 39.6 & (Martin, 1999a) \\
\hline & Poultry & Solomon Islands & 1998 & 54.0 & (Martin and Epstein, 1999) \\
\hline & Poultry & Tokelau & 1998 & 11.2 & (Martin, 1999b) \\
\hline & Poultry & Tonga & 1992-1994 & 36.4 & (Saville, 1996c) \\
\hline & Poultry & Tonga & 2010 & $+?$ & (으) \\
\hline & Poultry & Wallis \& Futuna & 1997-1998 & 5.7 & (Martin, 1999c) \\
\hline Murray valley encephalitis virus & Poultry & PNG & 2011 & +++ & (Schuster et al., 2011) \\
\hline \multirow[t]{4}{*}{ Newcastle disease } & Poultry & Cook Islands & 1993-1994 & 15.4 & (Saville, 1994) \\
\hline & Poultry & Kiribati & 1992-1994 & 56.2 & (Saville, 1996a) \\
\hline & Poultry & Tonga & 1992-1994 & 41.6 & (Saville, 1996c) \\
\hline & Poultry & Tonga & 2010 & $+?$ & (OIE) \\
\hline \multirow[t]{4}{*}{ Parvovirus } & Dog & Kiribati & 1992-1994 & + & (Saville, 1996a) \\
\hline & Dog & Samoa & 1997 & 80.0 & (Martin, 1999a) \\
\hline & Dog & Tonga & 1992-1994 & + & (Saville, 1996c) \\
\hline & Dog & Wallis \& Futuna & 1997-1998 & 25.0 & (Martin, 1999c) \\
\hline Disease & Species & Country & Date & Key result ${ }^{\mathrm{a}}$ & Reference \\
\hline \multirow[t]{5}{*}{ Parvovirus (Cont') } & Pig & Cook Islands & 1993-1994 & 25.0 & (Saville, 1994) \\
\hline & Pig & Guam & 1999 & 50.0 & (Duguies et al., 2000) \\
\hline & Pig & Kiribati & 1992-1994 & 10.0 & (Saville, 1996a) \\
\hline & Pig & Tokelau & 1998 & 97.7 & (Martin, 1999b) \\
\hline & Pig & Wallis \& Futuna & 1997-1998 & + & (Martin, 1999c) \\
\hline Porcine respiratory and reproductive syndrome & Pig & French Polynesia & 2006-2007,2011 & + & (으) \\
\hline \multirow[t]{4}{*}{ Q fever } & Cattle & Guam & 1999 & 1.4 & (Duguies et al., 2000) \\
\hline & Goat & Guam & 1998 & 8.6 & (Duguies et al., 2000) \\
\hline & Goat & Solomon Islands & 1998 & 3.1 & (Martin and Epstein, 1999) \\
\hline & Multi species & Vanuatu & 2011 & $+?$ & (을 \\
\hline \multirow[t]{2}{*}{ Ross River virus } & Multi species & Oceania & 1995 & +++ & (Sammels et al., 1995) \\
\hline & Multi species & PNG & 2001 & +++ & (Harley et al., 2001) \\
\hline Rotavirus & Pig & Solomon Islands & 1998 & 71.4 & (Martin and Epstein, 1999) \\
\hline \multirow[t]{3}{*}{ Sacbrood virus } & Bee & Niue & 1992, 1994 & + & (Saville, 1996b) \\
\hline & Bee & Samoa & 1996 & + & $(\underline{S P C}, 2004 d)$ \\
\hline & Bee & Solomon Islands & 1993 & 7.9 & (Reid and Van Eaton, 1993) \\
\hline Transmissible gastroenteritis & Pig & French Polynesia & 2011 & + & ( $\underline{\text { OIE }}$ \\
\hline Turkey rhinotracheitis & Poultry & French Polynesia & 2006-2007 & + & (OIE) \\
\hline \multicolumn{6}{|l|}{ PARASITIC DISEASES } \\
\hline Amoeba disease & Bee & Niue & 1992, 1994 & + & (Saville, 1996b) \\
\hline \multirow[t]{2}{*}{ Ancylostomiasis } & Cat & New Caledonia & 1993 & + & (Beugnet and Gadat, 1993) \\
\hline & Dog & New Caledonia & 1993 & + & (Beugnet and Gadat, 1993) \\
\hline \multirow[t]{8}{*}{ Babesiosis } & Cattle & French Polynesia & $2005-2007,2011$ & + & (OIE) \\
\hline & Cattle & New Caledonia & 2007 & + & (Barre et al., 2011) \\
\hline & Cattle & New Caledonia & 2008 & + & (Martin, 2009) \\
\hline & Cattle & New Caledonia & 2011 & + & (OIE) \\
\hline & Cattle & Samoa & 1997 & 8.5 & (Martin, 1999a) \\
\hline & Cattle & Samoa & 2008 & + & (OIE) \\
\hline & Cattle & Solomon Islands & 1998 & 6.9 & (Martin and Epstein, 1999) \\
\hline & Cattle & Tonga & $1992-1994$ & 0.6 & (Saville, 1996c) \\
\hline Capillariasis & Dog & Samoa & $2010-2011$ & 2.0 & (Carslake et al., 2012) \\
\hline \multirow[t]{3}{*}{ Coccidiosis } & Poultry & Fiji & 2008 & + & ( $\underline{\mathrm{OIE}}$ \\
\hline & Poultry & French Polynesia & 2010 & + & (IE) \\
\hline & Poultry & New Caledonia & 2010 & + & (OIE) \\
\hline Contagious pustular dermatitis & Multi species & New Caledonia & 2009 & + & (을 \\
\hline Crocodylocapillaria longiovata & Crocodile & PNG & 1998 & + & (Moravec and Spratt, 1998) \\
\hline Dermatophilosis & Cattle & Guam & 1999 & + & (Duguies et al., 2000) \\
\hline & Goat & Cook Islands & 1993-1994 & + & (Saville, 1994) \\
\hline & Goat & Kiribati & 1992-1994 & 100.0 & (Saville, 1996a) \\
\hline Dipylidiasis & Dog & Samoa & 2010-2011 & 4.4 & (Carslake et al., 2012) \\
\hline & Dog & New Caledonia & 1993 & 57.0 & (Beugnet et al., 1993) \\
\hline & Dog & New Caledonia & 1994 & 50.3 & (Beugnet et al., 1994) \\
\hline & Dog & New Caledonia & 2009 & 22.4 & (Watier-Grillot et al., 2011) \\
\hline & Dog & Samoa & 1980 & +++ & (Samarawickrema et al., 1992) \\
\hline & Dog & Samoa & 2010-2011 & 46.8 & (Carslake et al., 2012) \\
\hline Ehrlichiosis & Dog & Samoa & 1997 & 60.0 & (Martin, 1999a) \\
\hline & Dog & Wallis \& Futuna & 1997-1998 & 71.4 & (Martin, 1999c) \\
\hline Fasciolosis & Cattle & Samoa & 1997 & + & (Martin, 1999a) \\
\hline Filariosis & Multi species & French Polynesia & 2010 & + & ( OIE) \\
\hline & Multi species & New Caledonia & 2010 & + & (OIE) \\
\hline Fleas & Dog & Samoa & 2010-2011 & 83.7 & (Carslake et al., 2012) \\
\hline
\end{tabular}




\begin{tabular}{|c|c|c|c|c|c|}
\hline \multirow[t]{4}{*}{ Gastrointestinal parasites } & Goat & Fiji & 1996 & + & (Manueli, 1996) \\
\hline & Goat & PNG & 2011 & 89.1 & (Koinari et al., 2012) \\
\hline & Sheep & Fiji & 1996 & + & (Manueli, 1996) \\
\hline & Sheep & PNG & 2011 & 71.8 & (Koinari et al., 2012) \\
\hline Giardiasis & Dog & Samoa & $2010-2011$ & 14.6 & (Carslake et al., 2012) \\
\hline Hippoboscidae & Pigeon & New Caledonia & 1996 & + & (Beugnet et al., 1996) \\
\hline Hookworm & Dog & Samoa & $2010-2011$ & 90.7 & (Carslake et al., 2012) \\
\hline Leishmaniosis & Dog & New Caledonia & 2011 & + & (OIE) \\
\hline Lice & Dog & Samoa & $2010-2011$ & 8.1 & (Carslake et al., 2012) \\
\hline Mallophaga lice species & Pigeon & New Caledonia & 1997 & + & (Beugnet et al., 1996) \\
\hline Mammomonogamus spp. & Cat & CNMI & 2008 & + & (Tudor et al., 2008) \\
\hline Mange & Sheep & New Caledonia & 2008 & + & (OIE) \\
\hline \multirow[t]{2}{*}{ Mites } & Bee & Niue & 1998 & + & $(\underline{\mathrm{SPC}, 2004 \mathrm{c}})$ \\
\hline & Bee & Samoa & 1996 & + & $(\overline{S P C,}, 2004 d)$ \\
\hline \multirow[t]{2}{*}{ Nematophagous fungi } & Goat & Fiji & 1999 & + & (Manueli et al., 1999) \\
\hline & Sheep & Fiji & 1999 & + & (Manueli et al., 1999) \\
\hline \multirow[t]{5}{*}{ Nosemosis } & Bee & Fiji & 2008 & + & (OIE) \\
\hline & Bee & New Caledonia & 2010 & + & $(\overline{\mathrm{OIE}})$ \\
\hline & Bee & Niue & 1992, 1994 & +++ & (Saville, 1996b) \\
\hline & Bee & Samoa & 1996 & + & $(\underline{S P C, 2004 d)}$ \\
\hline & Bee & Solomon Islands & 1993 & 31.0 & (Reid and Van Eaton, 1993) \\
\hline Pentastomid parasites & Crocodile & Oceania & 2006 & + & (Junker and Boomker, 2006) \\
\hline \multirow[t]{2}{*}{ Rhipicephalus spp. } & Cattle & French Polynesia & 2010 & + & (Barre and Uilenberg, 2010) \\
\hline & Cattle & New Caledonia & 1995 & +++ & (Beugnet and Chardonnet, 1995) \\
\hline Disease & Species & Country & Date & Key result ${ }^{a}$ & Reference \\
\hline \multirow[t]{3}{*}{ Rhipicephalus spp. (Cont') } & Cattle & New Caledonia & 2010 & + & (Barre and Uilenberg, 2010) \\
\hline & Cattle & New Caledonia & 2010 & + & (De Meeus et al., 2010) \\
\hline & Cattle & PNG & 2010 & + & (Barre and Uilenberg, 2010) \\
\hline Rickettsia africae & Bird & New Caledonia & 2001-2007 & + & (Eldin et al., 2011) \\
\hline Screwworm (C. Bezziana) & Multi species & PNG & 2011 & + & (OIE) \\
\hline Subcutaneous filarial worm & Pigeon & New Caledonia & 1996 & + & (Beugnet et al., 1996) \\
\hline \multirow[t]{7}{*}{ Theileriosis } & Cattle & New Caledonia & 2011 & + & (OIE) \\
\hline & Cattle & Palau & 1994 & 22.2 & (Saville, 1999) \\
\hline & Cattle & Samoa & 1997 & 82.6 & (Martin, 1999a) \\
\hline & Cattle & Samoa & 2008 & + & (OIE) \\
\hline & Cattle & Solomon Islands & 1998 & 16.5 & (Martin and Epstein, 1999) \\
\hline & Cattle & Tonga & 1992-1994 & 29.8 & (Saville, 1996c) \\
\hline & Cattle & Tonga & 2010 & 333.0 & (OIE) \\
\hline Ticks & Dog & Samoa & $2010-2011$ & 42.1 & (Carslake et al., 2012) \\
\hline \multirow[t]{3}{*}{ Toxocariasis } & Cat & New Caledonia & 1993 & + & (Beugnet and Gadat, 1993) \\
\hline & Dog & New Caledonia & 1993 & + & (Beugnet and Gadat, 1993) \\
\hline & Dog & Samoa & $2010-2011$ & 3.4 & (Carslake et al., 2012) \\
\hline \multirow[t]{15}{*}{ Toxoplasmosis } & Cat & New Caledonia & 2009 & 50.0 & (Roqueplo et al., 2011) \\
\hline & Cattle & New Caledonia & 2009 & 3.3 & (Roqueplo et al., 2011) \\
\hline & Deer & New Caledonia & 2009 & 13.8 & (Roqueplo et al., 2011) \\
\hline & Dog & New Caledonia & 2009 & 32.8 & (Roqueplo et al., 2011) \\
\hline & Goat & Cook Islands & 1993-1994 & 63.3 & (Saville, 1994) \\
\hline & Goat & Guam & 1998 & 51.6 & (Duguies et al., 2000) \\
\hline & Goat & Kiribati & 1992-1994 & 100.0 & (Saville, 1996a) \\
\hline & Goat & Niue & 1992, 1994 & 87.5 & (Saville, 1996b) \\
\hline & Goat & Palau & 1996 & 100.0 & (Saville, 1999) \\
\hline & Goat & Solomon Islands & 1998 & 84.4 & (Martin and Epstein, 1999) \\
\hline & Goat & Tonga & 1992-1994 & 52.0 & (Saville, 1996c) \\
\hline & Horse & New Caledonia & 2009 & 16.0 & (Roqueplo et al., 2011) \\
\hline & Multi species & Fiji & 2008 & + & ( \\
\hline & Multi species & New Caledonia & 2008 & + & $(\underline{\mathrm{OIE}})$ \\
\hline & Pig & New Caledonia & 2009 & 2.0 & (Roqueplo et al., 2011) \\
\hline \multirow[t]{14}{*}{ Trichinellosis } & Crocodile & PNG & 2004 & 11.1 & (Pozio et al., 2004) \\
\hline & Crocodile & PNG & 2005 & 21.2 & (Pozio et al., 2005) \\
\hline & Pig & Cook Islands & 1993-1994 & 25.4 & (Saville, 1994) \\
\hline & Pig & Fiji & 2001 & + & (Reid et al., 2001) \\
\hline & Pig & Kiribati & 2001 & + & (Reid et al., 2001) \\
\hline & Pig & Kiribati & 2011 & + & ( \\
\hline & Pig & Kiribati & 1992-1994 & 44.7 & (Saville, 1996a) \\
\hline & Pig & Palau & 1996 & 1.7 & (Saville, 1999) \\
\hline & Pig & PNG & $1988-1998$ & + & (Pozio et al., 1999) \\
\hline & Pig & PNG & 2000 & + & (Owen et al., 2000) \\
\hline & Pig & PNG & 2001 & +++ & (Reid et al., 2001) \\
\hline & Pig & PNG & 2005 & 12.3 & (Pozio et al., 2005) \\
\hline & Pig & PNG & 2011 & + & (OIE) \\
\hline & Pig & Samoa & 1997 & 6.7 & (Martin, 1999a) \\
\hline
\end{tabular}




\begin{tabular}{|c|c|c|c|c|c|c|}
\hline & & Pig & Solomon Islands & 1998 & 1.6 & (Martin and Epstein, 1999) \\
\hline & & Pig & Tonga & 2010 & 333.0 & (OIE) \\
\hline & & Pig & Wallis \& Futuna & 1997-1998 & 0.6 & (Martin, 1999c) \\
\hline \multicolumn{2}{|c|}{ Trichomonosis } & Multi species & New Caledonia & $2005-2007,2011$ & + & (OIE) \\
\hline \multicolumn{2}{|c|}{ Trichophyton verrucosum } & Deer & New Caledonia & 1994 & +++ & (Lebel and Beugnet, 1994) \\
\hline \multicolumn{2}{|c|}{ Trichuris spp. } & Dog & Samoa & $2010-2011$ & 6.9 & (Carslake et al., 2012) \\
\hline \multicolumn{2}{|c|}{ Tropilaelaps infestation } & Bee & PNG & 2011 & + & (OIE) \\
\hline \multirow{4}{*}{\multicolumn{2}{|c|}{ Trypanosomosis }} & Dog & PNG & 1998 & + & (Reid and Copeman, 2003) \\
\hline & & Goat & PNG & 1998 & + & (Reid and Copeman, 2003) \\
\hline & & Horse & PNG & 1998 & + & (Reid and Copeman, 2003) \\
\hline & & Pig & PNG & 1998 & + & (Reid and Copeman, 2003) \\
\hline \multicolumn{2}{|c|}{ Varroasis } & Bee & PNG & 2011 & + & (OIE) \\
\hline \multicolumn{7}{|c|}{ OTHER DISEASES } \\
\hline \multirow{2}{*}{\multicolumn{2}{|c|}{ Chalkbrood }} & Bee & Samoa & 1996 & + & (SPC, 2004d) \\
\hline & & Bee & Tonga & 1991 & + & $(\underline{S P C}, 2004 \mathrm{e})$ \\
\hline \multicolumn{2}{|c|}{ Equine leucoencephalomalacia } & Horse & New Caledonia & 1996 & + & (Le Bars and Le Bars, 1996) \\
\hline \multirow{2}{*}{\multicolumn{2}{|c|}{ Half moon disorder }} & Bee & Samoa & 1996 & + & $(\underline{S P C}, 2004 d)$ \\
\hline & & Bee & Solomon Islands & 1993 & + & (Reid and Van Eaton, 1993) \\
\hline \multicolumn{2}{|c|}{ Heat-stable enterotoxin II-producing E. coli } & Pig & New Caledonia & 1994 & + & (Germani et al., 1994) \\
\hline \multicolumn{2}{|c|}{ Shiga-like toxin I-producing E. Coli } & Cattle & New Caledonia & 1994 & + & (Germani et al., 1994) \\
\hline \multirow{3}{*}{\multicolumn{2}{|c|}{ Wax moth }} & Bee & Niue & 1992, 1994 & + & (Saville, 1996b) \\
\hline & & Bee & Samoa & 1996 & + & $(\overline{S P C}, 2004 d)$ \\
\hline & & Bee & Solomon Islands & 1993 & + & (Reid and Van Eaton, 1993) \\
\hline \multicolumn{7}{|c|}{$\begin{array}{l}\text { Estimated prevalence in } \% \\
\text { +: Disease clinically observed, det } \\
\text { +++: Disease enzootic or very com } \\
\text { +? : Disease suspected to be prese }\end{array}$} \\
\hline
\end{tabular}

disease said to be common, very common or enzootic $(+++)$; diseases being suspected but not confirmed (+?).

A comparison of diseases or pathogens listed in Table 2 and 3 shows that 16 diseases were sought but could not be found using the described methodology, in the Pacific Islands region. These diseases included borreliosis, psittacosis, bovine ephemeral fever, canine distemper virus, classical swine fever, equine infectious anaemia, equine viral arteritis, foot and mouth disease, porcine rotavirus, simbu serogroup, swine influenza, vesicular stomatitis, Amblyomma spp., avian malaria, cryptosporidiosis and cysticercosis (however, since this literature review focuses exclusively on domestic animals, it cannot be ruled out that some may occur in wildlife reservoirs). Two recent literature reviews on parasitic zoonoses and parasites of animals recorded at the National Veterinary Laboratory in PNG were retrieved but the extensive lists of parasites presented in these papers could not be included in the Table 2 (Owen, 2005, 2011). No references were retrieved between 1992 and 2012 for five of the 22 PICTs included in this review, namely: American Samoa, Marshall Islands, Nauru, Pitcairn Islands and Tuvalu. Among the 77 references from 1992 to 2012, 31 (40.3\%) were from the grayliterature retrieved from the SPC local database.

\section{Discussion}

\subsection{Data and language limitations}

While the original objective of our work was to conduct a meta analysis, we found that too few up-to-date studies were available to do so. Therefore, the work presented in this paper resulted in being a descriptive review of the data available on domestic animal diseases in the Pacific Islands region. The only similar kind of work performed is an annotated 
Table 3

Animal diseases not detected or reported as not present in the Pacific Islands region based on the selected references from 1992 to 2012

\begin{tabular}{|c|c|c|c|c|c|c|c|c|c|c|c|c|c|c|c|}
\hline Animal diseases & Cook Islands $^{\mathrm{a}}$ & $\mathrm{Fiji}^{\mathrm{b}}$ & Guam $^{\mathrm{c}}$ & Kiribati $^{d}$ & $\begin{array}{c}\text { New } \\
\text { Caledonia }^{\mathrm{e}} \\
\end{array}$ & Niue $^{f}$ & Oceania $^{\mathrm{g}}$ & Palau $^{\text {h }}$ & $\mathrm{PNG}^{\mathrm{i}}$ & Samoa $a^{j}$ & $\begin{array}{l}\text { Solomon } \\
\text { Islands }^{k}\end{array}$ & Tokelau' & Tonga $^{\mathrm{m}}$ & Vanuatu $^{n}$ & $\begin{array}{l}\text { Wallis \& } \\
\text { Futuna }\end{array}$ \\
\hline \multicolumn{16}{|c|}{ Bacterial diseases } \\
\hline \multicolumn{16}{|l|}{ Anaplasmosis } \\
\hline Cattle & 1993 & & & & & & & 1995 & & & & & 1992 & & \\
\hline \multicolumn{16}{|c|}{ Avian encephalomyelitis } \\
\hline Poultry & & & & & & 1991 & & & & & & & & & \\
\hline \multicolumn{16}{|l|}{ Bartonellosis } \\
\hline Dog & & & & & 2009 & & & & & & & & & & \\
\hline Horse & & & & & 2009 & & & & & & & & & & \\
\hline \multicolumn{16}{|l|}{ Borreliosis } \\
\hline Dog & & & & & & & & & & 2010 & & & & & \\
\hline \multicolumn{16}{|l|}{ Brucellosis } \\
\hline Carabao & & & 1999 & & & & & & & & & & & & \\
\hline Cattle & 1993 & & 1999 & & & 1992 & & 1994 & & & 1998 & & 1992 & & \\
\hline Dog & & & & & & & & & & 1997 & & & & & 1997 \\
\hline Goat & 1993 & & 1998 & & & 1992 & & 1996 & & & 1998 & & 1992 & & 1997 \\
\hline Pig & 1993 & & 1999 & 1992 & & 1994 & & 1996 & & 1997 & 1998 & 1998 & & & 1997 \\
\hline \multicolumn{16}{|c|}{ Enzootic pneumonia } \\
\hline Pig & & & 1999 & & & & & & & & & & & & \\
\hline \multicolumn{16}{|c|}{ European foulbrood } \\
\hline Bee & & & & & & & & & & & 1993 & & & & \\
\hline \multicolumn{16}{|l|}{ Leptospirosis } \\
\hline Cat & & 2002 & & & & & & & & & & & & & \\
\hline Cattle & & & 1999 & & & 1992 & & & 2006 & & & & & & \\
\hline Goat & & & & & & & & & & & & & & & 1997 \\
\hline Horse & & & & & & & & & 2006 & & & & & & \\
\hline Pig & & & 1999 & 1992 & & & & & & & & & & & \\
\hline \multicolumn{16}{|c|}{ Paratuberculosis (Johne's disease) } \\
\hline Cattle & & & 1999 & & & 1992 & & & & 1997 & & & & & \\
\hline Goat & 1993 & & & & & & & & & & 1998 & & & & 1997 \\
\hline \multicolumn{16}{|l|}{ Psittacosis } \\
\hline Poultry & & & & & & 1991 & & & & & & & & & \\
\hline \multicolumn{16}{|l|}{ Salmonellosis } \\
\hline \multirow{2}{*}{\multicolumn{16}{|c|}{ Tuberculosis }} \\
\hline & & & & & & & & & & & & & & & \\
\hline Carabao & & & 1999 & & & & & & & & & & & & \\
\hline Cattle & 1993 & & 1999 & & & 1992 & & 1994 & & & 1998 & & 1992 & & \\
\hline Goat & 1993 & & 1998 & & & & & & & & & & & & \\
\hline \multicolumn{16}{|l|}{ Viral diseases } \\
\hline \multicolumn{16}{|l|}{ Akabane virus } \\
\hline Cattle & 1993 & & & & & & & & & & & & 1992 & & \\
\hline \multicolumn{16}{|c|}{ Aujeszky's disease (Pseudorabies) } \\
\hline Pig & 1993 & & 1999 & 1992 & & 1994 & & 1996 & & & 1998 & & & & \\
\hline \multicolumn{16}{|c|}{ Avian infectious laryngotracheitis } \\
\hline \multirow{2}{*}{\multicolumn{16}{|c|}{$\begin{array}{l}\text { Poultry } \\
\text { Avian influenza }\end{array}$}} \\
\hline & & & & & & & & & & & & & & & \\
\hline Poultry & 1993 & & 1999 & 1992 & & 1991 & 2003 & 1996 & & 1997 & 1998 & 1998 & 1992 & & 1997 \\
\hline
\end{tabular}




\begin{tabular}{|c|c|c|c|c|c|c|c|c|c|c|c|c|c|c|c|}
\hline Animal diseases & Cook Islands ${ }^{a}$ & $\mathrm{Fiji}^{\mathrm{b}}$ & Guam $^{\mathrm{C}}$ & Kiribati $^{d}$ & $\begin{array}{c}\text { New } \\
\text { Caledonia }\end{array}$ & Nive $^{f}$ & Oceania $^{\mathrm{g}}$ & Palau $^{\text {h }}$ & $\mathrm{PNG}^{\prime}$ & Samoaj & $\begin{array}{l}\text { Solomon } \\
\text { Islands }^{k}\end{array}$ & Tokelau' & Tonga $^{\mathrm{m}}$ & Vanuatu $^{n}$ & $\begin{array}{l}\text { Wallis \& } \\
\text { Futuna }^{\circ}\end{array}$ \\
\hline \multicolumn{16}{|l|}{ Bluetongue } \\
\hline Carabao & & & 1999 & & & & & & & & & & & & \\
\hline Cattle & 1993 & & & & & 1992 & & 1994 & & 1997 & & & 1992 & & \\
\hline Goat & 1993 & & 1998 & 1992 & & 1992 & & 1996 & & & 1998 & & 1992 & & 1997 \\
\hline \multicolumn{16}{|c|}{$\begin{array}{l}\text { Goat } \\
\text { Bovine ephemeral fever }\end{array}$} \\
\hline Cattle & 1993 & & & & & 1992 & & 1995 & & & & & 1992 & & \\
\hline \multicolumn{16}{|c|}{ Bovine virus diarrhea } \\
\hline Cattle & & & & & & 1992 & & 1994 & & & 1998 & & & & \\
\hline \multicolumn{16}{|c|}{ Canine distemper virus } \\
\hline \multicolumn{16}{|c|}{$\begin{array}{l}\text { Dog } \\
\text { Caprine arthritis encephalitis }\end{array}$} \\
\hline \multirow{2}{*}{\multicolumn{16}{|c|}{ Classical swine fever }} \\
\hline & & & & & & & & & & & & & & & \\
\hline \multicolumn{16}{|c|}{$\begin{array}{l}\text { Pig } \\
\text { Enzootic bovine leukosis }\end{array}$} \\
\hline \multirow{2}{*}{\multicolumn{16}{|c|}{ Equine infectious anaemia }} \\
\hline & & & & & & & & & & & & & & & \\
\hline Horse & 1993 & & 1999 & & & & & & & 1997 & 1998 & & 1992 & & 1997 \\
\hline \multicolumn{16}{|l|}{ Equine influenza } \\
\hline \multirow{2}{*}{\multicolumn{16}{|c|}{$\begin{array}{l}\text { Horse } \\
\text { Equine rhinopneumonitis }\end{array}$}} \\
\hline & & & & & & & & & & & & & & & \\
\hline \multirow{2}{*}{\multicolumn{16}{|c|}{$\begin{array}{l}\text { Horse } \\
\text { Equine viral arteritis }\end{array}$}} \\
\hline & & & & & & & & & & & & & & & \\
\hline \multirow{2}{*}{\multicolumn{16}{|c|}{ Foot and mouth disease }} \\
\hline & & & & & & & & & & & & & & & \\
\hline Cattle & 1993 & & & & & & & & & & & & & & \\
\hline Goat & 1993 & & & & & & & & & & & & & & \\
\hline Pig & 1993 & & & & & & & & & & & & & & \\
\hline \multicolumn{16}{|c|}{ Infectious bovine rhinotracheitis } \\
\hline Carabao & & & 1999 & & & & & & & & & & & & \\
\hline \multirow{2}{*}{\multicolumn{16}{|c|}{ Japanese encephalitis virus }} \\
\hline & & & & & & & & & & & & & & & \\
\hline Dog & & & & & & & & & & 1997 & & & & & 1997 \\
\hline Goat & & & & & & & & & & 1997 & & & & & 1997 \\
\hline Horse & & & 1999 & & & & & & & & & & & & 1997 \\
\hline Pig & & & & & & & & 1997 & & 1997 & & 1998 & & & 1997 \\
\hline \multicolumn{16}{|l|}{ Newcastle disease } \\
\hline Poultry & & 2000 & 1999 & & & 1991 & & 1996 & & 1997 & 1998 & 1998 & & & 1997 \\
\hline \multicolumn{16}{|c|}{$\begin{array}{l}\text { Porcine respiratory and } \\
\text { eproductive syndrome }\end{array}$} \\
\hline Pig & & & 1999 & & & & & 1996 & & 1997 & 1998 & 1998 & & & 1997 \\
\hline \multirow{2}{*}{\multicolumn{16}{|c|}{ Porcine rotavirus }} \\
\hline \multirow{2}{*}{\multicolumn{16}{|c|}{ Q fever }} \\
\hline & & & & & & & & & & & & & & & \\
\hline Carabao & & & 1999 & & & & & & & & & & & & \\
\hline Cattle & & & & & & 1992 & & & & & & & & & \\
\hline Goat & 1993 & & & 1992 & & 1992 & & 1996 & & & & & 1992 & & 1997 \\
\hline
\end{tabular}




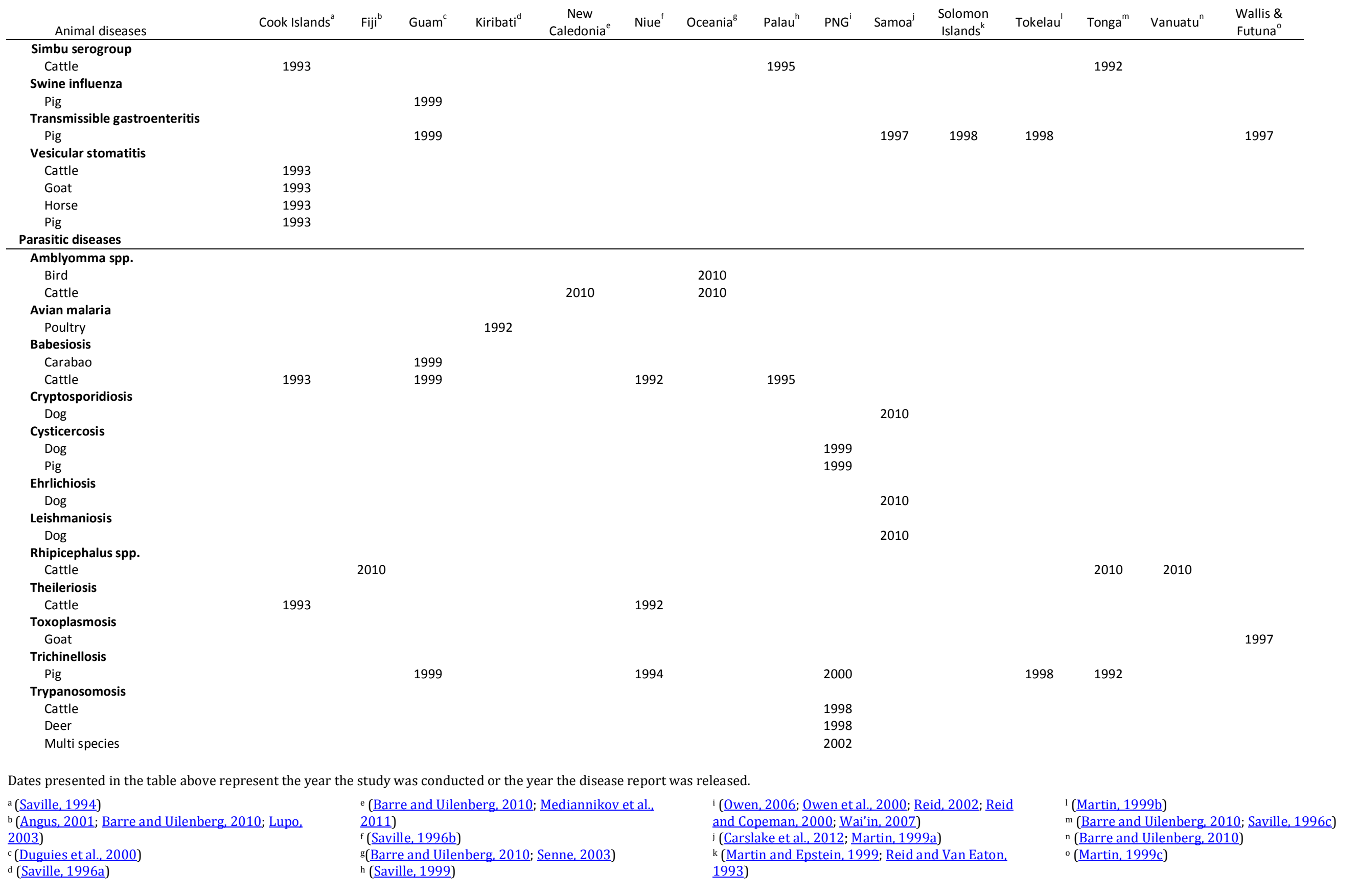


bibliography on animal husbandry and diseases in the Pacific area dating from 1966 (Pacific Science Information Center Bernice P. Bishop Museum Honolulu, 1966). The work presented in this present paper may help fill the gap in knowledge of animal diseases for the region and should give a wider access to currently available data.

Thanks to the access to the database available at the Secretariat for the Pacific Community and because we were able to review papers written either in English or in French (which are the 2 main official languages in the region), we think that we have been able to retrieve and analyse the majority and the most relevant references available on domestic animal diseases in the Pacific Islands region. It is acknowledged that the PICTs may have individually conducted animal disease studies without publishing the results on the databases selected for this study. This data would thus be missing in this literature review.

This review has extracted data from gray literature usually only accessible to a limited number of stakeholders within the Pacific Islands region and made this available to the scientific community.

However, the possibility of intentional non-reporting or public disclosure of animal diseases for fear of the negative impact on trade and tourism shall not be excluded.

The type of data collected varied between papers and reports. Some studies presenting only one piece of information for a particular animal disease in one species and for only one PICT while other studies gave an extensive list of data for several animal diseases studied in multiple species. In particular, SPC has conducted a series of animal disease surveys in 10 PICTs between 1994 and 2000 that provide an extensive amount of information for the main animal diseases in the region. However these data are already about 15 years old and would benefit from updated methods of disease detection.

The large number of references providing information for PNG and New Caledonia presumably reflects the interest and the investment in terms of animal disease surveillance and investigation done in these two countries by Australia and France respectively. This literature review shows that for 17 other PICTs less than five references were retrieved per country, emphasising the lack of information or lack of diseases in these countries.

The fact that a majority of data compiled focused on parasitic diseases may reflect the particular interest in such diseases in this part of the world and/or may indicate that these diseases are a main issue faced by local farmers. But it may also reflect the fact that few PICTs are actually equipped with diagnostic laboratories and most of the PICTs have to send samples abroad for a proper laboratory diagnosis of bacterial or viral diseases.

Our literature review shows that there is no clearly defined focus on a specific disease in the Pacific Islands region and that the research done so far in the region does not seem to highlight any one disease of particular significance to a single PICT or the region generally.

Most of the published data was related to cattle and pigs and this may reflect the importance of these two species for the livestock industry in the region but also the very particular role of pigs in Pacific society for traditional and social events (SPC, 2007). However, the proportionally limited amount of data retrieved for poultry is surprising considering the importance of that sector in most of the PICTs at the commercial and semicommercial level but also at the village level as a subsistence product for locals. 


\subsection{Diseases of zoonotic interest to the region}

In the literature reviewed, all samples tested for avian influenza were reported to be negative. The studies did not specify which strains were tested but our database query assessed whether there had been signs of pathology and/or high mortality in the local poultry population if a virulent strain was circulating in any of the PICTs. Only a low pathogenic strain of avian influenza was suspected in PNG in 2011 (OIE, 2011a).

Leptospirosis is an endemic zoonosis in Oceania (Kline et al., 2013) and is consistently reported as present in animals by the OIE member countries. The latest sero-prevalence studies on animals are from 2002 in Fiji (Lupo, 2003) and 2006 in PNG (Wai' in, 2007). Recent public health studies have been conducted in American Samoa and have been looking at the risk factors related to backyard piggeries (Lau et al., 2012a; Lau and DePasquale, 2012; Lau et al., 2012b). More studies within the susceptible animal populations and extended to the other PICTs are required to complement these studies in American Samoa.

Animal disease reports submitted for the year 2011 to OIE confirmed the present of Brucellosis in Fiji and French Polynesia and suspicion of occurrence in Tonga. The only recent sero-prevalence study retrieved for this disease was conducted in Wallis and Futuna islands (Antras and Garin-Bastuji, 2011).

Domestic animals are known to be the hosts of some arboviral infections such as Japanese encephalistis, Murray Valley encephalitis and Ross River virus (Kuno, 2001; Russell, 2002; Sabchareon and Yoksan, 1998) but only very limited information on these diseases was retrieved for domestic animals while they are identified as being of major importance in Oceania from a public health perspective (Kline et al., 2013).

\subsection{Based on the references retrieved through this literature search, rabies has not been detected or reported on domestic animals during the reference period of 1992 and this status seems to be confirmed by the "no" to "low" risk for humans of contracting rabies within the PICTs (WHO, 2008, 2011). But rabies was nevertheless reported as "present" in the Oceania region in 2007 (WHO, 2007). Diseases of economical importance and at potential risk for the region:}

Foot and Mouth disease and Classical Swine Fever are reported in various countries of South East Asia but PICTs seem to have remained free of these transboundary diseases. Similarly, Porcine respiratory and reproductive syndrome is now widely spreading in various parts of Asia (An et al., 2011), but within our PICT survey seems only to have been detected in French Polynesia. (OIE, 2011a). The Pacific Islands region therefore appears to be relatively free of economically important diseases. In an area composed of 25,000 islands dispersed over 180 million square kilometres and hosting 9 million people it is a challenge to maintain a disease free environment. So far, the Pacific region is said to have a "favourable animal health status" with almost no serious livestock diseases. Nevertheless, these statements have to be mitigated by the fact that many PICTs do not have adequate animal disease surveillance and reporting systems to confirm this status (Secretariat of the Pacific Community, 2009a, $\underline{b}$ ). However, an AusAID funded project is currently supporting the establishment of a network of laboratories in the region to help addressing this issue (REF???). 
Besides giving the PICTs the opportunity to access international markets, establishing and maintaining a national animal disease surveillance and information management system would create in-country benefits by enabling early detection of disease outbreaks and emerging diseases and reduce the impact of endemic diseases (Secretariat of the Pacific Community, 2009a)

\section{Conclusion}

This paper reviews the current knowledge on domestic animal diseases in 22 PICTs with an emphasis on data from 1992 to 2012 and shows that very little information is available for this region. While our review was seeking information on any domestic animal disease, no single disease appears to be a principal concern for the region. Considering the very broad scope of this review in terms of animal diseases, retrieved literature is scarce and no longer up-to-date. This paper stresses the need for more investment on animal disease status in Pacific Islands region, particularly given the tropical environment and ideal conditions for disease emergence. Responsible, commensurate investments and international coordination are needed to improve the knowledge of the current animal health status in the region and to enable PICTs wishing to control diseases of public health concern or to access international trade in live animals and animal products.

\section{Acknowledgements}

This review was conducted in partnership between the Animal Health and Production Team, Land and Resources Department of the Secretariat for the Pacific Community (SPC) and the School of Veterinary and Biomedical Sciences of James Cook University (JCU), Townsville, Queensland, Australia as part of the Food Animal Biosecurty Network project funded by the Australian Agency for International Development (AusAID).

We are most grateful to the Secretariat for the Pacific Community who provided access to their databases.

\section{References}

An, T.Q., Tian, Z.J., Leng, C.L., Peng, J.M., Tong, G.Z., 2011. Highly pathogenic porcine reproductive and respiratory syndrome virus, Asia. Emerg Infect Dis 17, 1782-1784.

Angus, S.D., 1986. Epidemiology and control of Helminth infections of domestic livestock on Pacific atolls, Master of Science in Tropical Veterinary Medicine - Center for Tropical Veterinary Medicine University of Edinburgh, $75 \mathrm{pp}$.

Angus, S.D., 2001. A Survey to Determine the Status of the Fiji Islands Concerning Newcastle Disease, Volume 221 of SPC technical paper Secretariat of the Pacific Community. 24 pp.

Antras, V., Garin-Bastuji, B., 2011. La brucellose porcine a Wallis et Futuna, Bulletin Epidemiologique, Sante Animale et Alimentation, pp. 31-34.

Barre, N., Happold, J., Delathiere, J.M., Desoutter, D., Salery, M., de Vos, A., Marchal, C., Perrot, R., Grailles, M., Mortelecque, A., 2011. A campaign to eradicate bovine babesiosis from New Caledonia. Ticks Tick Borne Dis 2, 55-61.

Barre, N., Uilenberg, G., 2010. Spread of parasites transported with their hosts: case study of two species of cattle tick. Rev Sci Tech 29, 149-160, 135-147. 
Beugnet, F., Bimablum, S., Chardonnet, L., 1993. Epidemiologic-study of dog dirofilariasis in NewCaledonia - Choice of a diagnosis methodology. Revue De Medecine Veterinaire 144, 891-897.

Beugnet, F., Chardonnet, L., 1995. Tick resistance to pyrethroids in New Caledonia. Veterinary Parasitology 56, 325-338.

Beugnet, F., Gadat, R., 1993. Survey on Toxocara spp eggs and Ancylostoma spp larvae in soil extracts from Noumea, New-Caledonia. Revue De Medecine Veterinaire 144, 523-525.

Beugnet, F., Gadat, R., Chardonnet, L., 1996. Parasitologic note: Parasites of Notu (Ducula goliath, Columbiform). About some observations. Recueil De Medecine Veterinaire 172, 421-424.

Beugnet, F., Rous, V., Leurs, M., Chardonnet, L., 1994. Age in cardiopulmonary Dirofilariasis of dog - Consequences on chemoprophylaxis. Revue De Medecine Veterinaire 145, 59-64.

Carslake, R.J., Hill, K.E., Sjolander, K., Prattley, D., Acke, E., 2012. Prevalence of selected infectious diseases in the Samoan dogs. Journal of Veterinary Internal Medicine 26, 788-788.

De Meeus, T., Koffi, B.B., Barre, N., de Garine-Wichatitsky, M., Chevillon, C., 2010. Swift sympatric adaptation of a species of cattle tick to a new deer host in New Caledonia. Infect Genet Evol 10, 976-983.

Desvars, A., Cardinale, E., Michault, A., 2011. Animal leptospirosis in small tropical areas. Epidemiol Infect 139, 167-188.

Duguies, M., Nusbaum, S., Saville, P., 2000. ADAP Animal Health Survey for Guam 1999. American Samoa Community College, College of Micronesia, Northern Marianas College, University of Guam, and University of Hawaii., 24 pp.

Eldin, C., Mediannikov, O., Davoust, B., Cabre, O., Barre, N., Raoult, D., Parola, P., 2011. Emergence of Rickettsia africae, Oceania. Emerg Infect Dis 17, 100-102.

Fukunaga, Y., Kumanomido, T., Kamada, M., 2000. Getah virus as an equine pathogen. Vet Clin North Am Equine Pract 16, 605-617.

Garin, B., Gouali, M., Wouafo, M., Perchec, A.-M., Pham Minh, T., Ravaonindrina, N., Urbes, F., Gay, M., Diawara, A., Leclercq, A., Rocourt, J., Pouillot, R., 2012. Prevalence, quantification and antimicrobial resistance of Campylobacter spp. on chicken neck-skins at points of slaughter in 5 major cities located on 4 continents. International Journal of Food Microbiology 157, $102-$ 107.

Germani, Y., Morillon, M., Begaud, E., Dubourdieu, H., Costa, R., Thevenon, J., 1994. Two-year study of endemic enteric pathogens associated with acute diarrhea in New Caledonia. J Clin Microbiol 32, 1532-1536.

Gummow, B., 2010. Challenges posed by new and re-emerging infectious diseases in livestock production, wildlife and humans. Livestock Science, 130 (1-3). pp. 41-46.

Harley, D., Sleigh, A., Ritchie, S., 2001. Ross River virus transmission, infection, and disease: a cross-disciplinary review. Clin Microbiol Rev 14, 909-932.

Hide, R., 2003. Pig husbandry in New Guinea. A literature review and bibliography.ACIAR Monograph No. 108, xvi + 291 pp.

Huchzermeyer, F.W., Langelet, E., Putterill, J.F., 2008. An outbreak of chlamydiosis in farmed Indopacific crocodiles (Crocodylus porosus). J S Afr Vet Assoc 79, 99-100.

Jones, K.E., Patel, N.G., Levy, M.A., Storeygard, A., Balk, D., Gittleman, J.L., Daszak, P., 2008. Global trends in emerging infectious diseases. Nature 451, 990-993.

Junker, K., Boomker, J., 2006. Check-list of the pentastomid parasites crocodilians and freshwater chelonians. Onderstepoort J Vet Res 73, 27-36.

Kaba, M., Davoust, B., Cabre, O., Colson, P., 2011. Hepatitis E virus genotype $3 \mathrm{f}$ in pigs in New Caledonia. Aust Vet J 89, 496-499.

Kline, K., McCarthy, J.S., Pearson, M., Loukas, A., Hotez, P.J., 2013. Neglected tropical diseases of Oceania: review of their prevalence, distribution, and opportunities for control. Plos Neglected Tropical Diseases 7, e1755.

Koinari, M., Karl, S., Ryan, U., Lymbery, A.J., 2012. Infection levels of gastrointestinal parasites in sheep and goats in Papua New Guinea. Journal of Helminthology, 1-7.

Kuno, G., 2001. Persistence of arboviruses and antiviral antibodies in vertebrate hosts: its occurrence and impacts $\uparrow$. Rev Med Virol 11, 165-190.Lau, C.L., Clements, A.C., Skelly, C., Dobson, A.J., Smythe, L.D., Weinstein, P., 2012a. Leptospirosis in American Samoa-- 
estimating and mapping risk using environmental data. Plos Neglected Tropical Diseases 6, e1669.

Lau, C.L., DePasquale, J.M., 2012. Leptospirosis, diagnostic challenges, American Samoa. Emerging Infectious Diseases 18, 2079-2081.

Lau, C.L., Dobson, A.J., Smythe, L.D., Fearnley, E.J., Skelly, C., Clements, A.C., Craig, S.B., Fuimaono, S.D., Weinstein, P., 2012b. Leptospirosis in American Samoa 2010: epidemiology, environmental drivers, and the management of emergence. The American Journal of Tropical Medicine and Hygiene 86, 309-319.

Le Bars, J., Le Bars, P., 1996. Recent acute and subacute mycotoxicoses recognized in France. Vet Res 27, 383-394.

Lebel, S., Beugnet, F., 1994. Ringworm cases on rusa deer in New-Caledonia. Revue De Medecine Veterinaire 145, 721-721.

Lupo, C., 2003. Epidémiologie de la leptospirose animale aux îles Fidji. Thèse d'exercice, Ecole Nationale Vétérinaire de Toulouse - ENVT, 2003, 151 pp.

Manueli, P.R.; Le Jambre, L.F.; Know, M.R., 1996. Conference Paper Date: 22-25 April, 1996, Conference Location: Bogor, Indonesia Pages: 92-97 Publisher: Australian Centre for International Agricultural Research (ACIAR) Canberra, Australia.

Manueli, P.R., Waller, P.J., Faedo, M., Mahommed, F., 1999. Biological control of nematode parasites of livestock in Fiji: screening of fresh dung of small ruminants for the presence of nematophagous fungi. Vet Parasitol 81, 39-45.

Martin, S., 2009. Bovine Babesiosis in New Caledonia, Report of the first FAO/OIE subregional meeting of the GF-TADs for the Pacific Community region in collaboration with SPC. Secretariat of the Pacific Community, Nadi, Fiji Islands, 48 pp.

Martin, T., 1999a. The animal health status of Samoa. Secretariat of the Pacific Community, Noumea (New Caledonia), 34 pp.Martin, T., 1999b. The animal health status of Tokelau Secretariat of the Pacific Community, Noumea (New Caledonia) 17 pp.

Martin, T., 1999c. The animal health status of Wallis \& Futuna. Secretariat of the Pacific Community, Noumea (New Caledonia) 29 pp.

Martin, T., Epstein, V., 1999. The animal health status of the Solomon Islands. Secretariat of the Pacific Community, Noumea (New Caledonia) $34 \mathrm{pp}$.

Mediannikov, O., Davoust, B., Cabre, O., Rolain, J.M., Raoult, D., 2011. Bartonellae in animals and vectors in New Caledonia. Comp Immunol Microbiol Infect Dis 34, 497-501.

Moravec, F., Spratt, D.M., 1998. Crocodylocapillaria longiovata n. gen., n. sp. (Nematoda: Capillariidae) from the stomach of crocodiles in Australia and New Guinea. J Parasitol 84, 426430.

Newman, S., McKenzie, A., 1991. Organisation of veterinary public health in Australasia and the Pacific Islands. Revue Scientifique et Technique 10, 1159-1184.

OIE, World Animal Health Information Database (WAHID) Interface. http://www.oie.int/wahis/public.php (accessed 30 January 2013).

OIE, 2011a. Animal health situation in Oceania. World Animal Health Information Database (WAHID) Interface.

http://www.oie.int/wahis_2/public/wahid.php/Countryinformation/Animalsituation (accessed 30 January 2013).

OIE, 2011b. Terrestrial animal health code, General provisions, pp. 435.

Owen, I.L., 2005. Parasitic zoonoses in Papua New Guinea. J Helminthol 79, 1-14.

Owen, I.L., 2006. Current status of Taenia solium and cysticercosis in Papua New Guinea. Parasitol Int 55 Suppl, S149-153.

Owen, I.L., 2011. Parasites of animals in Papua New Guinea recorded at the National Veterinary Laboratory: a catalogue, historical review and zoogeographical affiliations. Zootaxa, 1-163.

Owen, I.L., Sims, L.D., Wigglesworth, M.C., Puana, I., 2000. Trichinellosis in Papua New Guinea. Aust Vet J 78, 698-701.

Pacific Science Information Center Bernice P. Bishop Museum Honolulu, H., 1966. Annotated bibliography on animal husbandry and diseases in the Pacific area, South Pacific Comission Technical meeting on livestock production and health, Suva, FIJI. 
Paterson, R.A., Robertson, I.D., Sanders, R.C., Siba, P.M., Clegg, A., Hampson, D.J., 1993. The carriage of Streptococcus suis type 2 by pigs in Papua New Guinea. Epidemiol Infect 110, 7178.

Pozio, E., Owen, I.L., La Rosa, G., Sacchi, L., Rossi, P., Corona, S., 1999. Trichinella papuae n.sp. (Nematoda), a new non-encapsulated species from domestic and sylvatic swine of Papua New Guinea. Int J Parasitol 29, 1825-1839.

Pozio, E., Owen, I.L., Marucci, G., La Rosa, G., 2004. Trichinella papuae in saltwater crocodiles (Crocodylus porosus) of Papua New Guinea. Emerging Infectious Diseases 10, 1507-1509.

Pozio, E., Owen, I.L., Marucci, G., La Rosa, G., 2005. Inappropriate feeding practice favors the transmission of Trichinella papuae from wild pigs to saltwater crocodiles in Papua New Guinea. Vet Parasitol 127, 245-251.

Reid, G.M., Van Eaton, C., 1993. A survey of honey bee pests and diseases in the Solomon Islands. Ministry of External Relations and Trade, Wellington.

Reid, S., Puana, I., Kapo, N., Owen, I., Wai'in, P., Angus, S.D., Fenwick, S., Robertson, I., Smythe, L., 2001. The identification of constraints and possible remedies to livestock production by zoonotic diseases in the South Pacific. ACIAR, $47 \mathrm{pp}$.

Reid, S.A., 2002. Trypanosoma evansi control and containment in Australasia. Trends Parasitol 18, 219-224.

Reid, S.A., Copeman, D.B., 2000. Surveys in Papua New Guinea to detect the presence of Trypanosoma evansi infection. Aust Vet J 78, 843-845.

Reid, S.A., Copeman, D.B., 2003. The development and validation of an antibody-ELISA to detect Trypanosoma evansi infection in cattle in Australia and Papua New Guinea. Prev Vet Med 61, 195-208.

Roqueplo, C., Halos, L., Cabre, O., Davoust, B., 2011. Toxoplasma gondii in wild and domestic animals from New Caledonia. Parasite 18, 345-348.

Russell, R.C., 2002. Ross River virus: Ecology and distribution. Annual Review of Entomology 47, 1.

Sabchareon, A., Yoksan, S., 1998. Japanese encephalitis. Annals of Tropical Paediatrics 18, 5.

Samarawickrema, W.A., Kimura, E., Sones, F., Paulson, G.S., Cummings, R.F., 1992. Natural infections of Dirofilaria immitis in Aedes (Stegomyia) polynesiensis and Aedes (Finlaya) samoanus and their implication in human health in Samoa. Trans R Soc Trop Med Hyg 86, 187-188.

Sammels, L.M., Coelen, R.J., Lindsay, M.D., Mackenzie, J.S., 1995. Geographic distribution and evolution of Ross River virus in Australia and the Pacific Islands. Virology 212, 20-29.

Savidge, J.A., Sileo, L., Siegfried, L.M., 1992. Was disease involved in the decimation of Guam's avifauna? J Wildl Dis 28, 206-214.

Saville, P., 1994. The animal health status of the Cook Islands. Secretariat of the Pacific Community, Noumea (New Caledonia) $12 \mathrm{pp}$.

Saville, P., 1996a. The animal health status of Kiribati. Secretariat of the Pacific Community, Noumea (New Caledonia) 10 pp.

Saville, P., 1996b. The animal health status of Niue. Secretariat of the Pacific Community, Noumea (New Caledonia) $10 \mathrm{pp}$.

Saville, P., 1996c. The animal health status of Tonga. Secretariat of the Pacific Community, Noumea (New Caledonia) $18 \mathrm{pp}$.

Saville, P., 1999. The animal health status of Palau. Secretariat of the Pacific Community, Noumea (New Caledonia) 19 pp.

Schuster, G., Ebert, E.E., Stevenson, M.A., Corner, R.J., Johansen, C.A., 2011. Application of satellite precipitation data to analyse and model arbovirus activity in the tropics. Int J Health Geogr 10, 8.

Secretariat of the Pacific Community, 2009a. Annual Report 2009, Part 1 - Annual overview.

Secretariat of the Pacific Community, 2009b. Report of the first FAO/OIE subregional meeting of GF-TADs for the Pacific Community region in collaboration with SPC.

Secretariat of the Pacific Community, 2004a. Apiculture in Cooks Islands, Country report and future strategy. Secretariat of the Pacific Community, Suva (Fiji), 9 pp. 
Secretariat of the Pacific Community, 2004b. Apiculture in Fiji, Country report and future strategy. Secretariat of the Pacific Community, Suva (Fiji), 12 pp.

Secretariat of the Pacific Community, 2004c. Apiculture in Niue, Country report and future strategy. Secretariat of the Pacific Community, Suva (Fiji), 11 pp.

Secretariat of the Pacific Community, 2004d. Apiculture in Samoa, Country report and future strategy. Secretariat of the Pacific Community, Suva (Fiji), 13 pp.

Secretariat of the Pacific Community, 2004e. Apiculture in Tonga, Country report and future strategy. Secretariat of the Pacific Community, Suva (Fiji), 11 pp.

Secretariat of the Pacific Community, 2004f. Brucella suis brucellosis outbreak in Wallis and Futuna. Inform'Action 18.

Secretariat of the Pacific Community, 2007. The Importance of the pig in Pacific Island Culture. An annotated bibliography. Secretariat of the Pacific Community, Suva (Fiji), 43 pp.

Senne, D.A., 2003. Avian influenza in the Western Hemisphere including the Pacific Islands and Australia. Avian diseases 47, 798-805.

Simms, J.R., 1998. Animal leptospirosis in the Federated States of Micronesia. Pacific Health Dialog $5,30-37$.

Thevenon, J., Rantoen, D., Carton, D., Costa, R., Trap, D., 1992. [Infection by avian chlamydiosis in breeding pigeons in New Caledonia]. Ann Rech Vet 23, 63-71.

Trott, D.J., Combs, B.G., Mikosza, A.S., Oxberry, S.L., Robertson, I.D., Passey, M., Taime, J., Sehuko, R., Alpers, M.P., Hampson, D.J., 1997. The prevalence of Serpulina pilosicoli in humans and domestic animals in the Eastern Highlands of Papua New Guinea. Epidemiol Infect 119, 369-379.

Trott, D.J., Mikosza, A.S., Combs, B.G., Oxberry, S.L., Hampson, D.J., 1998. Population genetic analysis of Serpulina pilosicoli and its molecular epidemiology in villages in the eastern Highlands of Papua New Guinea. Int J Syst Bacteriol 48 Pt 3, 659-668.

Tudor, E.G., Lee, A.C., Armato, D.G., Bowman, D.D., 2008. Mammomonogamus auris infection in the middle ear of a domestic cat in Saipan, Northern Mariana Islands, USA. J Feline Med Surg $10,501-504$.

Vilain, O., Thevenon, J., Costa, R., Rantoen, D., 1994. Serologic survey for antibodies to IBR IPV virus in cattle in New-Caledonia. Recueil De Medecine Veterinaire 170, 539-545.

Wai'in, P.M., 2007. Epidemiology of infection with Leptospira species in Livestock in Papua New Guinea, School of Biomedical and Veterinary Sciences Murdoch University Perth, 214 pp.

Watier-Grillot, S., Marie, J.L., Cabre, O., Davoust, B., 2011. Survey of Canine Dirofilaria immitis Infection in New Caledonia. Vet Med Int 2011, 380680.

WHO 2007. Presence/absence of rabies in 2007. http://www.who.int/rabies/Absence_Presence_Rabies_07_large.jpg (accessed 16 December 2013).

WHO 2008. Rabies, countries or areas at risk. http://www.who.int/rabies/Global_Rabies_ITH_2008.png (accessed 16 December 2013).

WHO 2011. Distribution of risk levels for humans contracting rabies, worldwide, 2011. http://www.who.int/rabies/Global_distribution_risk_humans_contracting_rabies_2011.png (accessed 16 December 2013).

Yarrow, R., 2008. Increasing veterinary capacity in the Pacific, Secretariat of the Pacific Community Third regional meeting of Heads of Agriculture and Forestry Services (HOAFS), 4 pp. 